\title{
ENUMERATIVE COMBINATORICS OF SIMPLICIAL AND CELL COMPLEXES: KIRCHHOFF AND TRENT TYPE THEOREMS
}

\author{
SYLVAIN E. CAPPELL AND EDWARD Y. MILLER
}

\begin{abstract}
This paper considers three separate matrices associated to graphs and (each dimension of) cell complexes. It relates all the coefficients of their respective characteristic polynomials to the geometric and combinatorial enumeration of three kinds of subobjects. The matrices are: the mesh matrix for integral d-cycles, the mesh matrix for integral d-boundaries, and the Kirchhoff matrix, i.e., the combinatorial Laplacian, for integral (d-1)-chains.

Trent's theorem states that the determinant of the mesh matrix on 1-cycles of a connected graph is equal to the number of spanning trees [29, 30. Here this theorem is extended to the mesh matrix on $d$ cycles in an arbitrary cell complex and, new even for graphs, to enumerative combinatorial interpretation of all of the coefficients of its characteristic polynomial. This last is well defined once a basis for the integral $d$-cycles is chosen. Additionally, a parallel result for the mesh matrix for integral d-boundaries is proved.

Kirchhoff's theorem states that the product of the non-zero eigenvalues of the Kirchhoff matrix, i.e., combinatorial Laplacian, for connected graphs equals $n$ times the number of spanning trees with $n$ the number of vertices. Lyons has generalized Kirchhoff's result on the product of the non-zero eigenvalues of the Kirchhoff or combinatorial Laplacian on (d-1)-chains to cell complexes for $d>1$ [18. The present analysis extends this to all coefficients of the characteristic polynomial.

An evaluation of the Reidemeister-Franz torsion of the cell complex with respect to its integral basis gives relations between these combinatorial invariants.

$\begin{array}{ll}\text { Section 1. } & \text { Introduction } \\ \text { Section 2. } & \text { Theorem on mesh matrix for } d \text { cycles: evaluation of its characteristic polynomial } \\ \text { Section 3. } & \text { Proof of the main theorem for mesh matrix for } d \text { cycles: theorem }[2.1 \\ \text { Section 4. } & \text { Alternative version of the squared covolume } \operatorname{det}\left(j_{V} \cdot j_{V}^{\star}\right), \text { cases } k \geq 0 \\ \text { Section 5. } & \text { The mesh matrix for } d \text { boundaries: evaluation of its characteristic polynomial } \\ \text { Section 6. } & \text { Proof of main theorem } 5.1 \text { for mesh matrix for } d \text { boundaries: theorem } \\ \text { Section 7: } & \text { Evaluation of all coefficients of mesh matrices for cycles and boundaries } \\ & \text { with respect to geometrically defined bases. } \\ \text { Section 8. } & \text { A Kirchhoff type theorem for cell complexes ( extending Lyons [18] }) \\ \text { Section 9. } & \text { Homology covolume squared via determinants of mesh matrices } \\ \text { Section 10. } & \text { Combinatorial identities via a computation of Reidemeister-Franz torsion } \\ \text { Section 11. } & \text { Weights, Variant Settings, and Examples } \\ \text { Section 12. } & \text { Enumerative combinatorics of the simplex ( extending Kalai [14] ) }\end{array}$
\end{abstract}

\section{Introduction}

This paper considers three separate matrices associated to graphs and (each dimension of) cell complexes. It relates all the coefficients of their respective characteristic polynomials to the geometric and combinatorial enumeration of three kinds of sub-objects. Their determinants and more generally their characteristic polynomials are interpreted in terms of correspondingly three different aspects of the enumerative combinatorics of the graph or cell complex (in each dimension).

The oldest such matrix, of the three studied here, is Kirchhoff's matrix, which is the combinatorial Laplacian $\Delta_{0}$ [17, 11] on 0-chains. For graphs, $\Delta_{0}$ is the degree matrix minus the adjacency matrix. For a connected graph with $n$ vertices, Kirchhoff showed that the product of the non-vanishing eigenvalues of $\Delta_{0}$ is equal to $n$ times the number of spanning trees. This interpretation of the product of the non-vanishing eigenvalues of the combinatorial Laplacian on (d-1)-chains was generalized to higher dimensional complexes by Lyons 
[18] as an explicit weighted sum over pairs of combinatorial objects which he called bases and cobases, and here (consistent with the classical terminology for graphs) are called (ddimensional) spanning forests and ((d-1)-dimensional) spanning coforests. In the restricted context of d-dimensional complexes which are rationally acyclic in dimensions less than $d$, parallel theorems to those of Lyons on the product of the eigenvalues of the Krichhoff matrix had earlier appeared in the work of Duval, Klivans, and Martin [12].

In the case of graphs the complete characteristic polynomial of the Kirchhoff matrix had been studied and each of its coefficients had been evaluated by an enumerative combinatorial formula, a sum over special subgraphs, forests with specified number of edges with associated weights [8, 9]. For cell complexes, analysis of the characteristic polynomials is developed here giving an analogous interpretation for all coefficients of the polynomial in each dimension.

The second type of matrix studied here is the higher dimensional analogue of the mesh matrix introduced by Trent [29, 30] to study enumerative combinatorics of graphs. It is based on the idea that the integral cycles have a natural pairing to the integers, yielding a mesh matrix once a basis is chosen. As shown by Trent, the determinant of the mesh matrix of a connected graph equals the number of spanning trees. Here this classical work is extended in two new directions. One extends the definition and results about the Trent matrix for graphs to simplicial or cell complexes, obtaining a mesh matrix in each dimension $d$ with determinant a sum over (d-dimensional) spanning forests with explicit weights. Next, we give a geometrical interpretation for each coefficient in the characteristic polynomial of the mesh matrix of graphs as well as in the higher dimensional context. The mesh matrix is defined for each choice of integral basis for the d-cycles; its determinant is independent of this basis, while the coefficients of other powers of $t$ in the characteristic polynomial depend on this choice. We see that in $\S 5$, the coefficient of each power of $t$ is shown to be sum over geometrical objects, (d-dimensional) $\mathrm{k}$ augmented spanning forests, with positive integer weights depending in a specified way on the chosen basis. This is of interest and novel even in the graph setting. Moreover, these weights are here given for cell complexes and graphs several topological and combinatorial interpretations in settings of geometrical interest.

Thirdly, the integral boundaries of chains in each dimension also have a natural pairing to the integers. Once an integral basis is chosen a matrix arises. Its determinant is the sum over (d-dimensional) spanning coforests with explicit positive integral weights which are studied below. Again each coefficient of powers of $t$ in the characteristic polynomial has an expression as a sum uniformly over (d-dimensional) k reduced coforests with certain positive integral weights.

The geometrical objects being enumerated with weights in the formulae for each of the three settings are thus different. Let $n_{d}=\operatorname{dim} C_{d}(X ; R), z_{d}=\operatorname{dim} Z_{d}(X ; R), b_{d}=$ $\operatorname{dim} B_{d}(X ; R)$, the dimensions of the $d$-chains, $d$-cycles, $d$-boundaries. For the reader's convenience, these are tabulated in the following table. 


\begin{tabular}{|c|c|c|c|c|}
\hline & \multicolumn{2}{|c|}{ for connected graphs $(d=1)$} & \multicolumn{2}{|c|}{ for cell complexes } \\
\hline $\begin{array}{l}\text { Matrix } \\
\text { Types }\end{array}$ & $\begin{array}{c}\text { product } \\
\text { non-vanishing } \\
\text { eigenvalues }\end{array}$ & $\begin{array}{l}\text { coefficient of } t^{L} \\
\text { of characteristic } \\
\text { polynomial }\end{array}$ & $\begin{array}{c}\text { product } \\
\text { non-vanishing } \\
\text { eigenvalues }\end{array}$ & $\begin{array}{l}\text { coefficient of } t^{L} \\
\text { of characteristic } \\
\text { polynomial }\end{array}$ \\
\hline & \multicolumn{4}{|c|}{ Geometrical objects summed over to yield algebraic invariant } \\
\hline $\begin{array}{c}\text { Kirchhoff's } \\
\text { Combinatorial } \\
\text { Laplacian } \\
\text { on }(d-1) \text {-chains } \\
n_{d-1} \times n_{d-1}\end{array}$ & $\begin{array}{c}\text { spanning } \\
\text { trees } \\
L=1\end{array}$ & $\begin{array}{c}\text { forests of size } \mathrm{m} \\
L=n_{0}-m\end{array}$ & $\begin{array}{l}\text { pairs, spanning } \\
\text { forest and } \\
\text { spanning } \\
\text { coforest in it } \\
L=n_{d-1}-b_{d-1}\end{array}$ & $\begin{array}{l}\text { pairs, forest } \\
\text { of size } \mathrm{m} \text { and } \\
\text { spanning } \\
\text { coforest in it } \\
L=n_{d-1}-m \\
0 \leq m \leq b_{d-1}\end{array}$ \\
\hline $\begin{array}{c}\text { Trent } \\
\text { Mesh matrix } \\
\text { on d-cycles } \\
z_{d} \times z_{d}\end{array}$ & $\begin{array}{c}\text { spanning } \\
\text { trees } \\
L=0\end{array}$ & $\begin{array}{c}\mathrm{k} \text { augmented } \\
\text { spanning forests } \\
L=k\end{array}$ & $\begin{array}{c}\text { spanning forests } \\
L=0\end{array}$ & $\begin{array}{c}\mathrm{k} \text { augmented } \\
\text { spanning forests } \\
L=k \\
0 \leq k<z_{d}\end{array}$ \\
\hline $\begin{array}{c}\text { Mesh matrix } \\
\text { on d-boundaries } \\
b_{d} \times b_{d}\end{array}$ & - & - & $\begin{array}{c}\text { spanning coforests } \\
L=0\end{array}$ & $\begin{array}{c}\mathrm{k} \text { reduced } \\
\text { spanning coforests } \\
L=k \\
0 \leq k<b_{d}\end{array}$ \\
\hline
\end{tabular}

In the Kirchhoff case the pairs, (forest, spanning coforest in it) are in dimensions (d,d-1) respectively; in the cycle mesh matrix case the $\mathrm{k}$ augmented spanning forest is in dimension $\mathrm{d}$; in the boundary mesh matrix case the $\mathrm{k}$ reduced spanning coforest is in dimension $\mathrm{d}$. A k augmented spanning forest is a subcomplex for which removing some choice of $\mathrm{k}$ d-dimensional cells yields a spanning forest; a k reduced spanning coforest is a subcomplex for which adding some choice of $\mathrm{k}$ d-dimensional cells yields a spanning coforest.

Additionally, for the d-cycles given a spanning forest there is a geometrically defined basis for the rational cycle group $Z_{d}(X ; Q)$; again for the d-boundaries given a spanning forest in dimension $d+1$ there is a rationally defined basis for the rational boundary group $B_{d}(X ; Q)$. Consequently, one obtains two types of geometrically defined mesh type matrices. In section 7 , in each of these cases the complete characteristic polynomial is evaluated combinatorially.

A spanning tree for a connected graph with $n$ vertices is a choice of $n-1$ edges which together carry no 1-cycles. More generally, even when the graph is not necessarily connected, a choice of spanning tree for each connected component is called a spanning forest. In this possibly disconnected context, Trent's theorem for graphs asserts that the determinant of the mesh matrix for cycles is the number of spanning forests [29, 30]. Correspondingly, the generalization to the complete characteristic polynomial below is expressed in terms of spanning forests and its extension, k-augmented spanning forests.

The mesh matrix of 1 cycles for a graph and more generally for $d$ cycles of a $C W$ complex $X$ is easily explained. The $d$ chains, $C_{d}(X ; Z)$ have a basis of oriented $d$ cells, and this yields a combinatorially defined pairing by setting $\left\langle\sigma_{j}, \sigma_{k}\right\rangle=0$ if the cells are different and +1 if $j=k$. By linearity this gives a pairing of integral chains

$$
<., .>: C_{d}(X ; Z) \times C_{d}(X ; Z) \rightarrow Z .
$$

By definition the integral $d$ cycles, $Z_{d}(X ; Z)$ are the kernel of the boundary mapping $\partial$ : $C_{d}(Z ; Z) \rightarrow C_{d-1}(X ; Z)$. Now including the $d$ cycles $Z_{d}(X ; Z) \subset C_{d}(X ; Z)$ the composite $Z_{d}(X ; Z) \times Z_{d}(X ; Z) \subset C_{d}(X ; Z) \times C_{d}(X ; Z) \rightarrow Z$ gives the pairing $<., .>$.

Taking a basis for the $d$ cycles, which is a free abelian group, say $\left\{z_{r}\right\}$, there is the associated mesh matrix on $d$ cycles

$$
M\left\{z_{r}\right\}=\left\{<z_{j}, z_{k}>\right\} .
$$


An analogous matrix is associated to the integral $d$ boundaries, $B_{d}(X ; Z)=$ image of $\partial$ : $C_{d+1}(X ; Z) \rightarrow C_{d}(X ; Z)$ giving a mesh matrix for $d$ boundaries, once a basis is chosen for the integral boundaries.

In this paper, the determinant of the mesh matrix on $d$-cycles is shown to be a simple positive integer weighted sum over what are called here "spanning forests" in a finite cell complex $X$, extending the classical notion of spanning forests for graphs. These spanning forests are subsets of $d$ cells maximal with respect to the property of carrying no $d$ cycles. This extends Trents's theorem to each dimension, say d, of a cell complex. Two distinct combinatorial evaluations of the weights are here provided.

There is a corresponding notion of $\mathrm{k}$ augmented spanning forest which are just spanning forests with $k$ added $d$ cells. Having picked a basis for the integral $d$-cycles, the mesh matrix on $d$-cycles is a well-defined integral valued $z_{d} \times z_{d}$ square matrix. It is shown that the $\left(z_{d}-k\right)^{t h}$ elementary symmetric function of the eigenvalues of of the mesh matrix for cycles is a sum over corresponding $\mathrm{k}$ augmented spanning forest with positive integral weights. These weights are given an explicit dependence on the basis of $d$-cycles chosen. This extends Trent's theorem to the complete characteristic polynomial of the mesh matrix for cycles. The weights are given in two quite different formats (but equal). This is of interest even in the graph, $d=1$, case. These definitions and results appear in $\$ 2$. ( The spanning forests are called bases in Lyons [18]. )

Additionally, the mesh matrix for $d$ boundaries has a parallel development for cell complexes; although it is trivial for graphs. Having chosen a basis for the integral boundaries, it is a well defined matrix. The determinant is obtained by a positively integer weighted sum over spanning coforests. Having picked a basis for the integral $d$-boundaries, the $\left(b_{d}-k\right)^{t h}$ elementary symmetric function of the eigenvalues of the mesh matrix for $d$ boundaries is shown to be a sum over $\mathrm{k}$ reduced spanning coforests with appropriate positive integral weights depending on the choice of basis. A k reduced spanning coforest is just a spanning coforest with $k$ cells removed. These definitions and results appear in \$5. Again two distinct evaluations of the weights are provided.

In section 7 , for each of two mesh matrix cases, the complete characteristic polynomial is evaluated combinatorially in yet another way, using a geometrically defined basis of the rational $d$ cycles and boundaries. This employs a fixed choice of a spanning forest in dimension $d$ and $d+1$ respectively.

In the Kirchhoff case and in the mesh cycle and mesh boundary case there is a formal analogy. The combinatorial Laplacian on $d$ chains, the mesh matrix for $d$-cycles, and the mesh matrix for $d$ boundaries each are represented by matrices of the form, $A \cdot A^{t}$. The Cauchy-Binet theorem applies as an algebraic step in the analysis of such $A \cdot A^{t}$. The nonzero eigenvalues of $A \cdot A^{t}$ are real, non-negative numbers, their positive square roots are often referred to as the singular values of the matrix $A$.

This algebraic procedure applied in the combinatorial Laplacian setting recapitulates the approach of Lyons who proved that the product of the non-zero eigenvalues of this Laplacian, acting on (d-1) chains, equals the a sum over (d dimensional) spanning forests and ( (d-1) dimensional) spanning coforests contained in them with positive integral weights in the terminology used here [bases and cobases in his treatment] [18]. Here this result is extended to the coefficients of the complete characteristic polynomial using a sum over pairs, a (d 
dimensional) forest and a $((d-1)$ dimensional $)$ spanning coforest in it. These results appear in $\S 8$.

There is a large and growing literature on the general topic of generalizations, analogues and applications of Kirchhoff type theorems. A small sample of relevant combinatorially oriented papers and monographs would include (1847) G. Kirchhoff [17], (1889) A. Cayley [7], (1935) H. Whitney [33], (1954) H. M. Trent [29], (1955) H. M. Trent [30], (1960) N. I . Biggs [2], (1961) A. Nerode and H. Shank [24], (1967) P. R. Bryant 44, (1971) W. K. Chen [10], (1971) H. Shank [28], (1976) S. B. Maurer [19], (1976) J.W. Moon [22], (1978) S. Chaiken and D. Kleitman [8], (1979) W. Tutte [31, (1982) S. A. Chaiken [9], (1983) G. Kalai [14], (1992) R. M. Adin [1], (2000) R. Kenyon [15], (2009) A. Duval, C. Klivans, and J. Martin [12], (2009) R. Lyons [18], (2009) A. Petersson, (2011) R. Kenyon[16], (2013) M. Catanzaro, V. Chernyak, and J. Klein [6], the items after 1983 in higher dimensional settings.

The determinants of the mesh matrices for $d$ cycles divided by that for $d$ boundaries is directly related to the determinant of a corresponding matrix for the homology. A computation of the Reidemeister-Franz torsion of the $C W$ complex $X$, yields interesting relations with the above combinatorial items. These definitions and combinatorial identities appear in \$10. A use of Reidemeister-Franz torsion in the combinatorial context also appears in the work of Catanzaro, Chernyak, Klein [5, 6].

In section $\$ 12$ the example of the standard simplex is considered, using and extending results of Kalai [14].

This paper emphasizes combinatorial identities. We note, however, that as the coefficients in the formulas produced are generally shown to be positive integers, a wealth of interesting inequalities, often involving simplified expressions, result as unstated corollaries.

In a future paper we will see how the Cauchy-Binet Theorem can be extended from the context of the determinant, which can be regarded as the trace of a one dimensional irreducible representation to the context of the trace of an arbitrary irreducible representation [32. By these means, additional combinatorial-geometric formulae can be derived for graphs and cell complexes.

\section{Theorem on mesh matrix for $d$ Cycles}

Let $X$ be a finite $\mathrm{CW}$-complex. Orient the cells. This data provides a basis for the integral $d$-chains, $C_{d}(X ; Z)$, a free abelian group. This basis is called the cellular basis. Let the boundary map be $\partial_{d}: C_{d}(X ; Z) \rightarrow C_{d-1}(X ; Z)$, and set the $d$-cycles $Z_{d}(X ; Z)=$ kernel of $\partial_{d}$, and $(d-1)$ boundaries $B_{d-1}(X ; Z)=$ image of $\partial_{d}$, and correspondingly for real coefficients $R$.

Define a natural integral valued pairing on $C_{d}(X ; Z)$ by

$$
\begin{aligned}
& <., .>: C_{d}(X ; Z) \times C_{d}(X ; Z) \rightarrow Z \\
& \left(\Sigma_{j} a_{j}\left[\sigma_{j}\right], \Sigma_{k} b_{k}\left[\sigma_{k}\right]\right) \mapsto \Sigma_{j} a_{j} \cdot b_{j}
\end{aligned}
$$

where $S_{d}=\left\{\left[\sigma_{j}\right]\right\}$ is the cellular basis in dimension $d$. That is, the elements of the cellular basis are of length one and orthogonal.

As above, let $n_{d}=\left|S_{d}\right|$ be the number of $d$-cells and $z_{d}, b_{d}$ be the ranks of the free abelian groups, $Z_{d}(X ; Z), B_{d}(X ; Z)$, of integral $d$ cycles, $d$ boundaries respectively. 
Introduce the mesh pairings on $d$ cycles and $d$ boundaries:

$$
\begin{aligned}
& \mu: Z_{d}(X ; Z) \times Z_{d}(X ; Z) \subset C_{d}(X ; Z) \times C_{d}(X ; Z) \stackrel{<. \rightarrow}{\rightarrow} Z \\
& \nu: B_{d}(X ; Z) \times B_{d}(X ; Z) \subset C_{d}(X ; Z) \times C_{d}(X ; Z) \stackrel{<.}{\rightarrow} .>
\end{aligned}
$$

Let $d \geq 1$ and fix an ordered basis, say $\left\{z_{r} \mid r=1, \cdots, z_{d}\right\}$ for the integral $d$ cycles $Z_{d}(X ; Z)$ and define the associated mesh matrix for $d$ cycles $M\left\{z_{r}\right\}$ a $z_{d} \times z_{d}$ integral valued matrix by

$$
M\left\{z_{r}\right\}_{i, j}=<z_{i}, z_{j}>.
$$

Similarly, fix an ordered basis, say $\left\{b_{s} \mid s=1, \cdots, b_{d}\right\}$ for the integral $d$ boundaries $B_{d}(X ; Z)$ and define the associated mesh matrix for $d$ boundaries $M\left\{b_{z}\right\}$, a $b_{d} \times b_{d}$ integral valued matrix by

$$
M\left\{b_{s}\right\}_{i, j}=<b_{i}, b_{j}>.
$$

In the case of $X=\Gamma, d=1$, a connected graph, the study of the mesh matrix for 1 cycles, i.e., circuits, was initiated by Trent [29], who showed that in this case:

$$
\operatorname{det}\left(M\left\{z_{r}\right\}\right)=\# \text { of spanning/trees of the graph } \Gamma
$$

Of course, the determinant of $M\left\{z_{r}\right\}$ is the product of the eigenvalues of the real symmetric matrix $M\left\{z_{r}\right\}$.

Here Trent's analysis of the determinant is extended to each dimension of any finite CWcomplex and to the coefficients of the complete characteristic polynomial, $\operatorname{det}\left(t I d-M\left\{z_{r}\right\}\right)$ of the mesh matrix for $d$ cycles $M\left\{z_{r}\right\}$. Also, parallel results for $M\left\{b_{r}\right\}$, the mesh matrix for $d$ boundaries, will also be obtained.

Note that under change of basis of the $d$-cycles $z_{j} \mapsto \Sigma_{k} A_{j, k} z_{k}$ with $A \in G L\left(z_{d}, Z\right)$, the mesh matrix $M\left\{z_{r}\right\}$ changes to the mesh matrix to $A \cdot M\left\{z_{r}\right\} \cdot A^{t}$, with thus $\operatorname{det}\left(t I d-M\left\{z_{r}\right\}\right)$ changing to $\operatorname{det}\left(t I d-A \cdot M\left\{z_{r}\right\} \cdot A^{t}\right)$. ( It is only the determinant, $\operatorname{det}\left(M\left\{z_{r}\right\}\right)$ which is left invariant under all transformations $M\left\{z_{r}\right\} \mapsto A \cdot M\left\{z_{r}\right\} \cdot A^{t}$. This last holds since $A \in G L\left(z_{d}, Z\right)$ implies that $\operatorname{det}(A)= \pm 1$.) So it is remarkable that one can arrive at uniform geometric formulas for coefficients of the characteristic polynomial recording the dependence on the choice of integral basis used. This same observation also applies to $\operatorname{det}\left(t I d-M\left\{b_{r}\right\}\right)$, the characteristic matrix for the mesh matrix for $d$ boundaries.

Both mesh matrices have a useful decomposition: Let $A\left\{z_{r}\right\}, A\left\{b_{s}\right\}$, respectively, denote the integral valued $n_{d} \times z_{d}, n_{d} \times b_{d}$ matrices representing the inclusions

$$
i_{z}: Z_{d}(X ; Z) \subset C_{d}(X ; Z) \text { and } i_{b}: B_{d}(X ; Z) \subset C_{d}(X ; Z)
$$

with respect to the chosen integral cycle basis $\left\{z_{r}\right\}$ of $Z_{d}(X ; Z)$ and the chosen integral boundary basis $\left\{b_{s}\right\}$ of $B_{d}(X ; Z)$ and the cellular basis of $C_{d}(X ; Z)$.

Then the respective square $z_{r} \times z_{r}$ mesh matrix $M\left\{z_{r}\right\}$ and the $b_{d} \times b_{d}$ matrix $M\left\{b_{r}\right\}$ are given by:

$$
M\left\{z_{r}\right\}=A\left\{z_{r}\right\}^{t} \cdot A\left\{z_{r}\right\} \text { and } M\left\{b_{s}\right\}=A\left\{b_{s}\right\}^{t} \cdot A\left\{b_{s}\right\}
$$

More intrinsically, endow $C_{d}(X ; R), Z_{d}(X ; R), B_{d}(X ; Z)$ with the inner products for which the specified basis elements, cellular, d cycle, d boundary, respectively, have length one and are orthogonal. Denote by $\langle., .>,,<., .\rangle^{\#},<.,>^{\#_{b}}$ the respective inner products. 
ENUMERATIVE COMBINATORICS OF SIMPLICIAL AND CELL COMPLEXES: KIRCHHOFF AND TRENT TYPE THEOREM\$

Let $i_{z}^{\star}: C_{d}(X ; R) \rightarrow Z_{d}(X ; R)$ the adjoint of $i_{z}$, and $i_{b}^{\star}: C_{d}(X ; R) \rightarrow B_{d}(X ; R)$ the adjoint of $i_{b}$ be defined by :

$$
\begin{aligned}
& <i_{z}(a), b>=<a, i_{z}^{\star}(b)>^{\# z} \quad \text { for } a \in Z_{d}(X ; R), b \in C_{d}(X ; R) \\
& <i_{b}(a), b>=<a, i_{b}^{\star}(b)>^{\#} \text { for } a \in B_{d}(X ; R), b \in C_{d}(X ; R)
\end{aligned}
$$

Then the entries of $M\left\{z_{r}\right\}$ are just

$$
M\left\{z_{r}\right\}_{u, v}=<i_{z}\left(z_{u}\right), i_{z}\left(z_{v}\right)>=<z_{u}, i_{z}^{\star} \cdot i_{z}\left(z_{v}\right)>^{\#_{z}} .
$$

showing that the mesh matrix for $d$ cycles $M\left\{z_{r}\right\}$ is just the matrix representing the linear mapping: $i_{z}^{\star} \cdot i_{z}: Z_{d}(X ; R) \rightarrow Z_{d}(X ; R)$ and is here given in terms of $A\left\{z_{r}\right\}$ as described above. Similarly, the mesh matrix for $d$ boundaries $M\left\{b_{r}\right\}$ is just the matrix representing the linear mapping: $i_{b}^{\star} \cdot i_{b}: B_{d}(X ; R) \rightarrow B_{d}(X ; R)$ and is thus given in terms of $A\left\{b_{s}\right\}$ as described above.

Also, one may use the cellular basis, or equivalently the inner product $<$, , $>$, to define the adjoint $\partial_{d}^{\star}: C_{d-1}(X ; Z) \rightarrow C_{d}(X ; Z)$ of the boundary mapping on integral $d$ chains:

$$
\partial_{d}: C_{d}(X ; Z) \rightarrow C_{d-1}(X ; Z)
$$

If $K$ represent the boundary mapping in the cellular basis, the combinatorial Laplacian acting on (d-1)-chains is

$$
\Delta_{d-1}=\partial_{d} \cdot \partial_{d}^{\star}: C_{d-1}(X ; Z) \rightarrow C_{d-1}(X ; Z) .
$$

It is represented by the matrix $K \cdot K^{t}$. Hence, this setting has a parallel decomposition to that for the mesh matrices for cycles and boundaries. This differs slightly from the standard usage of this term in differential geometry, as explained in the next paragraph.

Note: Logically following differential geometry, one could for $d-1>0$ follow the convention that the Laplacian on $d-1$ chains is $\tilde{\Delta}_{d-1}=\partial_{d} \cdot \partial_{d}^{\star}+\partial_{d-1}^{\star} \partial_{d-1}$ where $\partial_{d-1}$ : $C_{d-1}(X ; R) \rightarrow C_{d-2}(X ; R)$. However, in view of the combinatorial Hodge decomposition, $C_{d-1}(X ; R)=\mathcal{H}_{d-1} \oplus\left\{\right.$ image $\left.\partial_{d}\right\} \oplus\left\{\right.$ image $\left.\partial_{d-1}^{\star}\right\}$, an orthogonal direct sum decomposition, the mapping $\partial_{d}^{\star}$ maps the eigenvectors of $\left(\partial_{d} \partial_{d}^{\star} \mid\left\{\right.\right.$ image $\left.\left.\partial_{d}\right\}\right)$ to eigenvectors of $\left(\partial_{d}^{\star} \partial_{d} \mid\left\{\right.\right.$ image $\left.\left.\partial_{d}^{\star}\right\}\right)$ with the same non-zero eigenvalues. Since the harmonics $\mathcal{H}_{d}$ are identified with $\left\{c \in C_{d}(X ; R) \mid \partial_{d}(c)=0, \partial_{d}^{\star}(c)=0\right\}$ and under the combinatorial deRham isomorphism map isomorphically to the $d^{t h}$ homology, $H_{d}(X ; R)$, to record the non-zero eigenvalues of $\tilde{\Delta}_{d-1}$ for all $d$ efficiently it suffices to record the non-zero eigenvalues of what is called here the combinatorial Laplacian $\Delta_{d-1}=\partial_{d} \cdot \partial_{d}^{\star}$ acting on (d-1)-chains. This observation was used in the work of D. Ray and I. M. Singer [26] on their analytic version of Reidemeister-Franz torsion.

In the context of a simple graph, say $\Gamma, d=1, C$ is just the familiar incidence matrix from edges to vertices. Moreover,

$$
\text { Kirchhoff matrix }=\text { Graph Laplacian } \Delta_{0} \text { on } 0-\text { chains }=C \cdot C^{t}=\partial_{1} \partial_{1}^{\star}=\text { Deg }- \text { Adj }
$$

where $A d j$ is the adjacency matrix with $A d j_{v, w}=+1$ if $\{v, w\}$ is an edge and $=0$ otherwise, while $D e g$ is the diagonal matrix with $D e g_{v, v}$ equal to the number of edges containing the vertex $v$.

For any subset $V \subset S_{d}$ of the $d$ cells, form the d dimensional subcomplex of $X$, called $X_{V}$ as follows:

$$
X_{V}=X^{(d-1)} \cup V
$$


where $X^{(d-1)}$ is the $d$-1-skeleton of $X$, the union of all cells of dimension at most $d-1$. By definition, $X_{V}$ has dimension $d$, with $|V|$ cells of dimension $d$, and the already oriented cells of $V$ provide an integral, real basis for $C_{d}\left(X_{V} ; Z\right), C_{d}\left(X_{V} ; R\right)$ respectively.

For $V \subset S_{d}$, let $V^{c}$ denote the complement of $V$ in $S_{d}, V^{c}=S_{d}-V$.

Now consider the boundary mapping

$$
\partial_{d}: C_{d}(X ; Z) \rightarrow C_{d-1}(X ; Z)
$$

with image the free group $B_{d-1}(X ; Z)$ of rank $b_{d-1}$. Suppose $b_{d-1}>0$. By adding successively one by one $d$-cells of $S_{d}$ to the $d-1$ skeleton of $X, X^{d-1}$, the rank the image under $\partial_{d}$ may be increased one by one until the maximal value $b_{d-1}$ is obtained. That is, one may exhibit subsets $V \subset S_{d}$ with

$$
|V|=b_{d-1} \text { and } C_{d}\left(X_{V}, R\right) \stackrel{\partial_{d}}{\rightarrow} B_{d-1}\left(X_{V} ; R\right) \subset B_{d-1}(X ; R) \text { an isomorphism. }
$$

In agreement with the graph case, one calls such a choice of $V \subset S_{d}$ with these two properties a spanning forest of dimension $d$ of $X$. The kernel of the composite for such $V$ is just $Z_{d}\left(X_{V} ; R\right)$, so alternatively, $V \subset S_{d}$ is a (d-dimensional) spanning forest precisely if

$$
|V|=b_{d-1} \text { and } Z_{d}\left(X_{V} ; R\right)=0 \text {. }
$$

The computation of the determinant of the mesh matrix in dimension $d, \operatorname{det}\left(M\left\{z_{r}\right\}\right)$, will be as a sum over spanning forests of dimension $d$ in $X$.

Correspondingly, given a spanning forest $V$, for each oriented d-cell, say $\sigma \in V^{c}$, the boundary $\partial_{d} \sigma$ lies in $B_{d-1}(X ; R)=B_{d-1}\left(X_{V} ; R\right)$ so is of the form $\partial_{d} c$ for a unique $c \in$ $C_{d}\left(X_{V} ; Q\right)$, by $V$ a spanning forest of $X$. In this way, a unique rational d-cycle $z[\sigma] \in$ $Z_{d}\left(X_{V \cup\{\sigma\}} ; Q\right)$ is defined such that its restriction to $\sigma$ has coefficient +1 . In particular, this shows that if $\left\{\sigma_{1}, \sigma_{2}, \cdots, \sigma_{k}\right\}$ are disjoint oriented cells of $S_{d}$ in $V^{c}$ [with necessarily $\left.k \leq\left|V^{c}\right|=n_{d}-b_{d-1}=z_{d}\right]$, the union

$$
W=V \cup\left\{\sigma_{1}, \sigma_{2}, \cdots, \sigma_{k}\right\}, 0 \leq k \leq z_{d}
$$

has the two properties

$$
|W|=b_{d-1}+k \text { and } Z_{d}\left(X_{W} ; R\right)=k \text {. }
$$

One calls such a set $W \subset S_{d}$ a $\mathbf{k}$ augmented spanning forest since it arises exactly by adding $\mathrm{k}$ d-cells to some (d-dimensional) spanning forest $V$. In particular, a spanning forest is just a 0 augmented spanning forest in this notation.

Recall of the short exact sequence,

$$
0 \rightarrow Z_{d}(X ; R) \subset C_{d}(X ; R) \stackrel{\partial_{d}}{\rightarrow} B_{d-1}(X ; R) \rightarrow 0 .
$$

Again, in view of this short exact sequence, an equivalent condition for $W$ to be a k augmented spanning forest is that $0 \leq k \leq z_{d}$ and

$$
\left|W^{c}\right|=z_{d}-k \text { and } j_{W}: Z_{d}(X ; R) \subset C_{d}(X ; R) \stackrel{\text { restrict }}{\rightarrow} C_{d}\left(X_{W^{c}} ; R\right) \text { is onto. }
$$

For example, if $W$ is a $\mathrm{k}$ augmented spanning forest of dimension $d$, then $\left|W^{c}\right|=n_{d}-$ $\left(b_{d-1}+k\right)=z_{d}-k$. Since the mapping from $Z_{d}(X ; R)$ to $C_{d}\left(X_{W^{c}} ; R\right)$ has kernel $Z_{d}(X ; R) \cap$ $C_{d}\left(X_{W} ; R\right)=Z_{d}\left(X_{W} ; R\right)$ of dimension $k$, the image of $Z_{d}(X ; R)$ in $C_{d}\left(X_{W^{c}} ; R\right)$ has dimension $z_{d}-k$. Since $\left|W^{c}\right|=z_{d}-k$, this implies that $Z_{d}(X ; R) \rightarrow C_{d}\left(X_{W^{c}} ; R\right)$ is onto, proving that the first definition implies the second. The converse follows in a similar manner. 
The computation of the coefficients of the characteristic polynomial of the mesh matrix for $d$ cycles of $M\left\{z_{r}\right\}$, will be in terms of a sum over (d-dimensional) k augmented spanning forests with suitable positive integral weights.

For a finitely generated abelian group $G$ let $[G]$ denote its torsion subgroup and $|[G]|$ the order of this subgroup.

For a finite cell complex $X$, let $t_{k}(X)$ denote the order of the torsion subgroup of the $k^{t h}$ homology $H_{k}(X ; Z)$, i.e. , $t_{k}(X)=\left|\left[H_{k}(X ; Z)\right]\right|$.

By definition, for $W$ a $\mathrm{k}$ augmented spanning forest, the mapping

$$
j_{W}: Z_{d}(X ; Z) \subset C_{d}(X ; Z) \rightarrow C_{d}\left(X, X_{W} ; Z\right)=C_{d}\left(X_{W^{c}} ; Z\right)
$$

is rationally onto. Now using the chosen cycle basis for $\left\{z_{r}\right\}$ for $Z_{d}(X ; Z)$ and the cellular basis for $C_{d}\left(X_{W^{c}} ; Z\right)$, the adjoint is a well defined injection

$$
j_{W}^{\star}: C_{d}\left(X_{W^{c}} ; Z\right) \rightarrow Z_{d}(X ; Z) .
$$

More formally, take the cellular metric $<., .>$ restricted to $C_{d}\left(X_{W^{c}} ; Z\right)$ and the cycle metric $<., .>^{\# z}$ on $Z_{d}(X ; Z)$ and form the adjoint. Hence, if $j_{W}$ is represented in these basis by the matrix, say $U_{W}$, an integer valued $\left(z_{d}-k\right) \times z_{d}$ matrix, then the adjoint is represented by $U_{W}^{\star}$, the transpose.

The determinant $\operatorname{det}\left(j_{W} \cdot j_{W}^{\star}\right)$ will be called the covolume squared of this inclusion $j_{W}^{\star}$ of the lattice. It may be seen to be equal to the square of the unit volume cell of the sublattice inside the real vector space $Z_{d}(X ; R)$ under the metric $<., .>^{\% z}$. In this case, as $U_{W}$ is an integral valued matrix, this covolume squared is a non-negative integer.

Similarly, one has the injection of $d$ cycles :

$$
m_{W}: Z_{d}\left(X_{W} ; Z\right) \subset Z_{d}(X ; Z)
$$

By $W$ a $\mathrm{k}$ augmented spanning forest the free abelian group $Z_{d}\left(X_{W} ; Z\right)$ has rank $k$. Pick an integral basis, say $\left\{\hat{z}_{u}\right\}_{u=1}^{k}$ of $Z_{d}\left(X_{W} ; Z\right)$. For this $d$ cycle basis and the already chosen standard basis on $\left\{z_{r}\right\}$ for $Z_{d}(X ; Z)$, let $B[W]$ be the $z_{d} \times k$ matrix representing this inclusion with respect to the integral basis already chosen. As seen the determinant

$$
\operatorname{det}\left(B[W]^{t} B[W]\right) \text { is a positive integer }
$$

and is independent of the choice of integral basis for $Z_{d}\left(X_{W} ; Z\right)$. It explicitly records the dependence on the fixed basis of $Z_{d}(X ; Z)$.

This determinant is employed in the first main theorem.

Theorem 2.1. The constant coefficient of $(-1)^{z_{d}} \operatorname{det}\left(t I d-M\left\{z_{r}\right\}\right)$ is

$$
\begin{aligned}
& \operatorname{det}\left(M\left\{z_{r}\right\}\right)=\text { product of eigenvalues of } M\left\{z_{r}\right\}=\sigma_{z_{d}}\left(M\left\{z_{r}\right\}\right) \\
& =\Sigma_{V} \text {, a spanning forest of dimension d }\left(\frac{t_{d-1}\left(X_{V}\right)}{t_{d-1}(X)}\right)^{2} .
\end{aligned}
$$

Also, for $V$, any spanning forest, i.e, a 0 augmented spanning forest, covolume squared of $\left(j_{V}^{\star}: C_{d}\left(X_{V^{c}} ; Z\right) \hookrightarrow Z_{d}(X ; Z)\right)=\operatorname{det}\left(j_{V} \cdot j_{V}^{\star}\right)=\left(\frac{t_{d-1}\left(X_{V}\right)}{t_{d-1}(X)}\right)^{2}$. 


$$
\begin{aligned}
& \text { If } 0<k<z_{d} \text {, then } \\
& \text { coef ficient of }(-1)^{z_{d}-k} t^{k} \text { in } \operatorname{det}\left(t \text { Id }-M\left\{z_{r}\right\}\right) \\
& =\left(z_{d}-k\right)^{\text {th }} \text { elementary symmetric function in eigenvalues of } M\left\{z_{r}\right\}=\sigma_{z_{d}-k}\left(M\left\{z_{r}\right\}\right) \\
& =\Sigma_{W, \text { a } k \text { augmented spanning forest }}\left(\frac{t_{d-1}\left(X_{W}\right)}{t_{d-1}(X)}\right)^{2} \operatorname{det}\left(B[W]^{t} B[W]\right) .
\end{aligned}
$$

Here the integral matrix $B[W]$ records, as above, the injection $m_{W}: Z_{d}\left(X_{W} ; Z\right) \subset Z_{d}(X ; Z)$. Moreover, for $W$, a $k$ augmented spanning forest, with $0 \leq k<z_{d}$

$$
\begin{aligned}
& \text { covolume squared of }\left(j_{W}^{\star}: C_{d}\left(X_{W^{c}} ; Z\right) \hookrightarrow Z_{d}(X ; Z)\right)=\operatorname{det}\left(j_{W} \cdot j_{W}^{\star}\right) \\
& =\left(\frac{t_{d-1}\left(X_{W}\right)}{t_{d-1}(X)}\right)^{2} \cdot \operatorname{det}\left(B[W]^{t} B[W]\right) .
\end{aligned}
$$

Additionally, the quotient $\frac{t_{d-1}\left(X_{W}\right)}{t_{d-1}(X)}$ is a positive integer for $W$ any $k$-augmented spanning forest; in particular, for $k=0$.

The first part extends Trent's theorem to cell complexes; the second extends it to evaluate the complete characteristic polynomial of the mesh matrix $M\left\{z_{r}\right\}$. Note that two separate expressions are given for the integer weights, one involving $X_{W}$ and the other $X_{W^{c}}$. The explicit dependence on the choice of the integer basis $\left\{z_{r}\right\}$ made for $Z_{d}(X ; Z)$ is recorded directly through $\operatorname{det}\left(B[W]^{t} B[W]\right)$.

In \$7, for a geometrically defined basis of $Z_{d}(X ; Q)$, given by a choice of $\mathrm{d}$ dimensional spanning forest, $V_{0}$, the coefficients of the complete characteristic polynomial of the cycle mesh matrix associated to that geometrical choice are given combinatorially. Similarly, in 97, for a $(\mathrm{d}+1)$ dimensional spanning forest $V_{1}$, there are geometrically defined bases for $B_{d}(X ; Q)$. Again in that section, the complete characteristic polynomial of the associated geometrically defined cycle and boundary mesh matrices are evaluated combinatorially.

\section{Proof of the main theorem for mesh matrix for $d$ cycles: Theorem 2.1}

In analyzing $M\left\{z_{r}\right\}=A\left\{z_{r}\right\}^{\star} \cdot A\left\{z_{r}\right\}$ and its characteristic polynomial the following elementary standard lemmas are helpful.

Let $A$ be a $m \times n$ matrix. For each pair $I, J$ of subsets $I \subset\{1,2, \cdots, m\}, J \subset\{1,2, \cdots, n\}$ let $A_{I, J}$ be the $|I| \times|J|$ minor of $A$ with row indices from the subset $I$ and column indices from the subset $J$.

Lemma 3.1. Let $A$ be square $n \times n$ matrix, then $\sigma_{k}(A)$ the $k^{\text {th }}$, elementary symmetric function of the eigenvalues, say $\lambda_{i}, i=1, \cdots, n$, counted with multiplicities, is given by the sum of determinants

$$
\sigma_{k}(A)=\Sigma_{I \subset\{1, \cdots, n\}} \text { with }|I|=k \quad \operatorname{det}\left(A_{I, I}\right)
$$

for $0<k \leq n$.

Lemma 3.2 (via Cauchy-Binet theorem.). Let $A, B$ be $m \times n, n \times m$ matrices respectively, then the non-vanishing eigenvalues of $A \cdot B, a m \times m$ matrix, and $B \cdot A, a n \times n$ matrix counted with multiplicities are identical and the $k^{\text {th }}$ elementary symmetric function of the 
ENUMERATIVE COMBINATORICS OF SIMPLICIAL AND CELL COMPLEXES: KIRCHHOFF AND TRENT TYPE THEOREM\$ eigenvalues of $A B$ or of $B A$ equals:

$$
\begin{aligned}
& \sigma_{k}(A B)=\Sigma_{I \subset\{1, \cdots, m\}} \text { with|I|=k} \operatorname{det}\left((A B)_{I, I}\right) \\
& =\Sigma_{I \subset\{1, \cdots, m\}} \text { and } J \subset\{1, \cdots, n\} \text { with }|I|=k,|J|=k \operatorname{det}\left(A_{I, J}\right) \operatorname{det}\left(B_{J, I}\right) \text { Cauchy-Binet } \\
& =\Sigma_{I \subset\{1, \cdots, m\}} \text { and } J \subset\{1, \cdots, n\} \text { with }|I|=k,|J|=k \operatorname{det}\left(B_{J, I}\right) \operatorname{det}\left(A_{I, J}\right) \\
& =\Sigma_{J \subset\{1, \cdots, n\}} \text { with }|J|=k \\
& =\sigma_{k}(A B)
\end{aligned}
$$

To prove the first lemma is elementary. The desired coefficient may be evaluated by differentiating $\operatorname{det}(t I d-A)$ a total of $(n-k)$ times and setting $t=0$. Due to the special form of the square matrix $(t I d-A)$, with $t^{\prime} s$ only down the diagonal, this gives a sum of determinants of minors along the diagonal blocks of size $(k \times k)=|I| \times|I|$ indexed by the sets $(I, I)$.

The second lemma follow from the first by utilizing, as in the statement of the theorem, the Cauchy-Binet theorem.

These lemmas can be applied to the mesh matrix on $d$ cycles $M\left\{z_{r}\right\}=A\left\{z_{r}\right\}^{t} \cdot A\left\{z_{r}\right\}$ for $d$ cycles where $A\left\{z_{r}\right\}$, a $n_{d} \times z_{d}$ integer valued matrix, represents the inclusion:

$$
i_{X}: Z_{d}(X ; Z) \subset C_{d}(X ; Z) .
$$

The rows of $A\left\{z_{r}\right\}$ are indexed by d-cells in $S_{d}$ and the columns by indices in $\left\{1, \cdots, z_{d}\right\}$ specifying elements of the cycle basis $\left\{z_{r}\right\}$ of $Z_{d}(X ; Z)$.

By the second lemma the $\left(z_{d}-k\right)^{t h}$ elementary symmetric function in the eigenvalues of $M\left\{z_{r}\right\}=A\left\{z_{r}\right\}^{t} \cdot A\left\{z_{r}\right\}$ equals the $\left(z_{d}-k\right)^{t h}$ elementary symmetric function in the eigenvalues of $A\left\{z_{r}\right\} \cdot A\left\{z_{r}\right\}^{t}$. By the first lemma this equals the sum of determinants,

$$
\Sigma_{I \subset S_{d} \text { with }|I|=z_{d}-k} \operatorname{det}\left(\left(A\left\{z_{r}\right\} \cdot A\left\{z_{r}\right\}^{t}\right)_{(I, I)}\right),
$$

where the sum, as indicated, is over the $d$-cells $S_{d}$ of $X$ which label the rows of $A\left\{z_{r}\right\}$.

Here there is the equalities of matrices,

$$
\begin{aligned}
& \left(A\left\{z_{r}\right\} \cdot A\left\{z_{r}\right\}^{t}\right)_{(I, I)}=\left(A\left\{z_{r}\right\}\right)_{\left(I,\left\{1, \cdots, z_{d}\right\}\right)} \cdot\left(A\left\{z_{r}\right\}^{t}\right)_{\left(\left\{1, \cdots, z_{d}\right\}, I\right)} \\
& =\left(A\left\{z_{r}\right\}\right)_{\left(I,\left\{1, \cdots, z_{d}\right\}\right)} \cdot\left(\left(A\left\{z_{r}\right\}\right)_{\left(I,\left\{1, \cdots, z_{d}\right\}\right)}\right)^{t},
\end{aligned}
$$

where $\left(A\left\{z_{r}\right\}\right)_{\left(I,\left\{1, \cdots, z_{d}\right\}\right)}$ represents

$$
r_{I}: Z_{d}(X ; Z) \subset C_{d}(X ; Z) \stackrel{\text { restrict }}{\rightarrow} C_{d}\left(X_{I} ; Z\right)
$$

and its transpose the adjoint for the cycle basis for $Z_{d}(X ; Z)$ and the cellular basis for $C_{d}\left(X_{I} ; Z\right)$. In toto, this proves:

The $\left(z_{d}-k\right)^{\text {th }}$ elementary symmetric function in the eigenvalues of $M\left\{z_{r}\right\}$ $=\Sigma_{I \subset S_{d}}$ with $|I|=z_{d}-k \operatorname{det}\left(r_{I} \cdot r_{I}^{\star}\right)$.

Now clearly, the determinant $\operatorname{det}\left(r_{I} \cdot r_{I}^{\star}\right)$ equals zero unless $r_{I}: Z_{d}(X ; Z) \subset C_{d}(X ; Z) \stackrel{\text { restrict }}{\rightarrow} C_{d}\left(X_{I} ; Z\right)$ is onto.

Here $|I|=z_{d}-k$, so this is precisely the condition that $I=W^{c}$ with $W$ a k augmented spanning forest. In this case,

$$
r_{I}=j_{W}: Z_{d}(X ; Z) \subset C_{d}(X ; Z) \stackrel{\text { restrict }}{\rightarrow} C_{d}\left(X_{W^{c}} ; Z\right) .
$$


That is, this proves:

$$
\begin{aligned}
& { }^{h} e\left(z_{d}-k\right)^{t h} \text { elementary symmetric function in the eigenvalues of } M\left\{z_{r}\right\} \\
& =\Sigma_{W \subset S_{d} \text { a } k \text { augmented spanning forest }} \operatorname{det}\left(j_{W} \cdot j_{W}^{\star}\right) \text {. }
\end{aligned}
$$

Here the adjoint of $j_{W}$ called here $j_{W}^{\star}$ is formed by declaring that the fixed chosen basis of $Z_{d}(X ; Z)$ is orthonormal and that the d-dimensional cellular basis of $C_{d}\left(X_{W^{c}} ; Z\right)$ is orthonormal.

To complete the proof of the theorem for the $k=0$ case, for a spanning forest, say $V$, one needs to show that the covolume squared is expressed as

Now consider

$$
\operatorname{det}\left(j_{V} \cdot j_{V}^{\star}\right)=\left(\frac{t_{d-1}\left(X_{V}\right)}{t_{d-1}(X)}\right)^{2}
$$

$$
j_{V}: Z_{d}(X ; Z) \rightarrow C_{d}\left(X_{V^{c}} ; Z\right) .
$$

It is a mapping of free abelian groups and is rationally an isomorphism by the definition of spanning forest, so $\operatorname{det}\left(j_{V} \cdot j_{V}^{\star}\right)$ is just the square of the order of the torsion abelian group $C_{d}\left(X_{V^{c}} ; Z\right) / j_{V}\left(Z_{d}(X ; Z)\right)$.

Now quite generally, let $W$ be a k-augmented spanning tree and consider the restriction mapping

$$
j_{W}: Z_{d}(X ; Z) \rightarrow C_{d}\left(X_{W^{c}} ; Z\right)
$$

which is rationally onto by $W$ an k-augmented spanning forest, it is claimed that the torsion group $C_{d}\left(X_{W^{c}} ; Z\right) / j_{V}\left(Z_{d}(X ; Z)\right)$ has order

$$
\left|C_{d}\left(X_{W^{c}} ; Z\right) / j_{W}\left(Z_{d}(X ; Z)\right)\right|=\frac{t_{d-1}\left(X_{W}\right)}{t_{d-1}(X)},
$$

in particular is an integer as asserted in theorem 2.1.

Hence, in the case $k=0$, this implies the desired formula above.

To see this last write $j_{W}$ as the composite

$$
j_{W}: Z_{d}(X ; Z) \subset C_{d}(X ; Z) \rightarrow C_{d}\left(X_{W^{c}} ; Z\right)=C_{d}\left(X, X_{W} ; Z\right)=Z_{d}\left(X, X_{W} ; Z\right)
$$

since $X$ and $X_{W}$ have the same (d-1) skeleton. Also note that $B_{d}(X ; Z)$ maps onto $B_{d}\left(X, X_{W} ; Z\right)$, so the desired quotient is isomorphic to the quotient

$$
H_{d}\left(X, X_{W} ; Z\right) /\left\{\text { image } H_{d}(X ; Z)\right\} \text {. }
$$

But recall from Lyons [18] the utility of introducing the long exact sequence for the pair $\left(X, X_{W}\right)$ which reads

$$
H_{d}(X ; Z) \stackrel{\alpha}{\rightarrow} H_{d}\left(X, X_{W} ; Z\right) \stackrel{\beta}{\rightarrow} H_{d-1}\left(X_{W} ; Z\right) \stackrel{\gamma}{\rightarrow} H_{d-1}(X ; Z) \rightarrow H_{d-1}\left(X, X_{W} ; Z\right)=0
$$

with $\alpha$ is rationally onto by $W$ a k augmented spanning forest.

Hence, letting $K$ denote the image of $\beta$ in $H_{d-1}\left(X_{W} ; Z\right)$, by exactness, the torsion group $C_{d}\left(X, X_{W} ; Z\right) / j_{V}\left(Z_{d}(X ; Z)\right) \cong H_{d}\left(X, X_{W} ; Z\right) /\left\{\right.$ image $\left.H_{d}(X ; Z)\right\}$ is isomorphic to image of $\beta=K$.

Now consider the associated mappings of torsion subgroups:

$$
\text { image } \beta=K=[K] \subset\left[H_{d-1}\left(X_{W} ; Z\right)\right] \stackrel{\gamma^{\prime}}{\rightarrow}\left[H_{d-1}(X ; Z)\right] \rightarrow 0
$$


By definition $\gamma^{\prime} \cdot \beta=0$. Now let $x$ be a torsion class in $H_{d-1}(X ; Z)$, say $N x=0$, with $N$ a positive integer. By exactness of the pair, there is $y \in H_{d-1}\left(X_{W} ; Z\right)$ with $\gamma(y)=x$. But by $\gamma(N y)=0, N y$ is in the image of $\beta$ which has only torsion image. In particular, $N y$ is torsion and so also is $y$. Hence, $\gamma^{\prime}$ is onto. Also, this shows the sequence is exact at $\left[H_{d-1}\left(X_{W} ; Z\right)\right]$. In toto, this proves the desired equality

$$
|K|=\left|\left[H_{d-1}\left(X_{W} ; Z\right)\right]\right| /\left|\left[H_{d-1}(X ; Z)\right]\right|=\frac{t_{d-1}\left(X_{W}\right)}{t_{d-1}(X)}=\text { a positive integer . }
$$

The remaining parts of theorem 2.1 will be addressed in the next section $\oiint 4$

\section{Alternative version of the squared covolume $\operatorname{det}\left(j_{W} \cdot j_{W}^{\star}\right)$, cases $k>0$}

To complete the proof of theorem 2.1, one must prove

$$
\text { squared covolume } \operatorname{det}\left(j_{W} \cdot j_{W}^{\star}\right)=\left(\frac{t_{d-1}\left(X_{W}\right)}{t_{d-1}(X)}\right)^{2} \operatorname{det}\left(B[W]^{t} B[W]\right)
$$

for $W$ a k-augmented spanning forest, $0<k<z_{d}$.

To prove this result the following algebraic lemma will play a crucial part. It is proved at the end of this section.

Lemma 4.1. Let $1 \leq u<n$ and $A$ be an invertible $n$ by $n$ matrix. Let $R$ be the $u \times n$ matrix obtained from $A$ by deleting the last $n-u$ rows of $A$. Let $S$ be the matrix obtained from $\left(A^{-1}\right)^{t}$, the transpose inverse of $A$ by deleting the first $u$ rows, then

$$
\operatorname{det}\left(R \cdot R^{t}\right)=\operatorname{det}(A)^{2} \cdot \operatorname{det}\left(S \cdot S^{t}\right) .
$$

To be more explicit, as before let $\left\{z_{r}\right\}$ be the fixed chosen basis for $Z_{d}(X ; Z)$, and

$$
i_{X}: Z_{d}(X ; Z) \subset C_{d}(X ; Z)
$$

be the inclusion. Use the cellular basis, $\left\{1 \cdot \sigma_{j} \mid j=1, \cdots, n_{d}\right\}$ of oriented d cells to define the $n_{d} \times z_{d}$ matrix $A$ by

$$
i_{X}\left(z_{r}\right)=\Sigma_{\sigma_{t} \in S_{d}} A_{t, r} \sigma_{t}
$$

\{Here, as is conventional, $\left.i_{X}\left(\Sigma_{r} L_{r} z_{r}\right)=\Sigma_{k}\left(\Sigma_{r} A_{k, r} L_{r}\right) \sigma_{k} \cdot\right\}$

Now let $W$ be a k-augmented spanning forest, say $W=V \cup I$, with $V$ a choice of spanning tree and $I \subset S_{d}-V$ of size $k$.

By $V$ a spanning tree, there is an isomorphism

$$
j_{V}: Z_{d}(X ; Q) \rightarrow C_{d}\left(X_{V^{c}} ; Q\right)
$$

of rational vector spaces.

Indeed, for each cell $\sigma_{j} \in V^{c}$ there is a unique rational d-cycle, say $z\left(\sigma_{j}\right)$ for $X$ which maps under $j_{V}$ to precisely the d-chain $1 \cdot \sigma_{j}$. That is,

$$
z\left(\sigma_{j}\right) \in Z_{d}\left(X_{V \cup \sigma_{j}} ; Q\right) \text { restricts to } 1 \cdot \sigma_{j}
$$

for each $\sigma_{j} \in V^{c}$. This geometrically defined cycle $z\left(\sigma_{j}\right), j=1, \cdots, z_{d}$, with $\sigma_{j}$ ranging over $V^{c}$, is uniquely determined by these requirements.

In this manner, having chosen the spanning forest $V$, one obtains a canonical rational geometric basis $\left\{z\left(\sigma_{j}\right) \mid \sigma_{j} \in V^{c}=S_{d}-V\right\}$ for $Z_{d}(X ; Q)$ which restricts under $j_{V}$ to precisely the cellular orthonormal basis of $C_{d}\left(X_{V^{c}} ; Q\right)$. Very nicely the associated natural geometric basis for $Z_{d}\left(X_{W} ; Q\right)$ with the same spanning forest $V \subset W$ is precisely $\left\{z\left(\sigma_{j}\right) \mid \sigma_{j} \in W-V\right\}$ 
and similarly $Z_{d}\left(X_{W^{c}} ; Q\right)$ has rational basis $\left\{z\left(\sigma_{j}\right) \mid \sigma_{j} \in W^{c}\right\}$. In this manner, this geometric basis respects the direct sum decomposition of $Z_{d}(X ; Q):\left[W=V \cup I, S_{d}-W=V^{c} \cup I\right.$ $|I|=k$.]

$$
Z_{d}(X ; Q)=Z_{d}\left(X_{W} ; Q\right) \oplus Z_{d}\left(X_{\left.V \cup W^{c}\right)} ; Q\right)=Z_{d}\left(X_{V \cup I} ; Q\right) \oplus Z_{d}\left(X_{\left.V \cup W^{c}\right)} ; Q\right)
$$

In particular the methods of lemma 4.1 apply for this choice of geometrical basis, those for $\sigma_{j} \in I$ and those with $\sigma_{j} \in W^{c}$.

As has been demonstrated above in terms of the restriction mapping $j_{X}: Z_{d}(X ; Z) \rightarrow$ $C_{d}\left(X_{V^{c}} ; Z\right)$ which is rationally an isomorphism with respect to an integral basis of $Z_{d}(X ; Z)$ to the cellular one has determinant $\pm \frac{t_{d-1}\left(X_{V}\right)}{t_{d-a}(X)}$. Alternatively expressed, the change of basis from the an integral basis of $Z_{d}(X ; Z)$ to the geometrical basis given by the spanning forest $V$ has determinant equal to $\pm \frac{t_{d-1}\left(X_{V}\right)}{t_{d-1}(X)}$. As observed above, the $k=0$ case of theorem 2.1 $\operatorname{det}(A)= \pm\left(\frac{t_{d-1}\left(X_{V}\right)}{t_{d-1}(X)}\right)$.

In a like manner, since $V$ as a spanning forest for $X_{W}$, the change of basis from the an integral basis of $Z_{d}\left(X_{W} ; Z\right)$ to the geometrical basis given by the spanning forest $V$ in $X_{W}$ for $Z_{d}(X ; Q)$ has determinant equal to $\pm \frac{t_{d-1}\left(X_{V}\right)}{t_{d-1}\left(X_{W}\right)}$.

Under this identification of $Z_{d}(X ; Q)$ with $C_{d}\left(X_{V^{c}} ; Q\right)$, the mapping $j_{V}$ becomes identified with the identity mapping

$$
I d: Z_{d}(X ; Q) \cong Z_{d}(X ; Q)
$$

with the the matrix $A$ becoming just the translation between the two bases $\left\{z_{r}\right\}$ and the geometric basis $z\left(\sigma_{j}\right)$ as in

$$
z_{r}=\Sigma_{1 \leq j \leq z_{d}} A_{j, r} z\left(\sigma_{j}\right)
$$

[As is seen by applying the isomorphism $j_{V}$ to both sides.]

Now arrange that the first $z_{d}-k$ cells of $V^{c}$ are those of $W^{c}=S_{d}-W=S_{d}-(V \cup I)=V^{c}-I$ a total of $z_{d}-k$ in all. Let $R$ be the $\left(z_{d}-k\right) \times z_{d}$ matrix obtained from $A$ by deleting the last $k$ rows. This matrix represents exactly the mapping

$$
j_{W}: Z_{d}(X ; Q) \stackrel{\text { restrict }}{\rightarrow} C_{d}\left(X_{W^{c}} ; Q\right) \cong Z_{d}\left(X_{V \cup W^{c}} ; Q\right)
$$

sending $z_{r} \mapsto \Sigma_{1 \leq j \leq z_{d}-k} A_{j, r} z\left(\sigma_{j}\right)$ [ as seen by applying $\left.j_{W}\right]$ and $\operatorname{det}\left(j_{W} \cdot j_{W}^{\star}\right)$ is represented by the matrix $R R^{\bar{T}}$.

On the other hand, inverting the matrix $A$, gives

$$
z\left(\sigma_{t}\right)=\Sigma_{r}\left(A^{-1}\right)_{r, t} z_{r}
$$

That is, the identify mapping

$$
I d: Z_{d}(X ; Q) \cong Z_{d}(X ; Q)
$$

which for the geometric basis on the domain, $z\left(\sigma_{j}\right)$ with $\sigma_{j} \in V^{c}$ and the fixed chosen basis $\left\{z_{r}\right\}$ on the range is represented by the matrix $A^{-1}$.

Hence, the inclusion of d-cycle spaces over the rationals

$$
i_{W}: Z_{d}\left(X_{W} ; Q\right) \subset Z_{d}(X ; Q)
$$

is represented for the domain by the geometrical basis $z\left(\sigma_{j}\right), 1 \leq j \leq k \sigma_{j} \in I$, and range basis $\left\{z_{r}\right\}$ by the matrix, say $S^{t}$, and is obtained from $A^{-1}$ by deleting the first $k$ columns. 
Equivalently, $i_{W}^{\star}$ is represented by $S$ which is obtained form $\left(A^{-1}\right)^{t}$ by deleting the first $k$ rows.

Since $B[W]$ by definition is the matrix for the inclusion $Z_{d}\left(X_{W} ; Z\right) \subset Z_{d}(X ; Z)$ with respect to the an integral basis for $Z_{d}\left(X_{W} ; Z\right)$ and the chosen basis $\left\{z_{r}\right\}$ for $Z_{d}(X ; Z)$, this observation shows that

$$
\operatorname{det}\left(B[W]^{t} \cdot B[W]\right)=\left(\frac{t_{d-1}\left(X_{V}\right)}{t_{d-a}\left(X_{W}\right)}\right)^{2} \cdot \operatorname{det}\left(i_{W}^{\star} \cdot i_{W}\right)
$$

and $i_{W} \cdot i_{W}^{\star}$ is represented by

$$
\begin{aligned}
& \left.\left(\left.\left(A^{-1}\right)_{r, t}^{t}\right|_{1 \leq t \leq z_{d}-k}\right) \cdot\left(A^{-1}\right)_{r^{\prime}, t^{\prime}}\right|_{1 \leq t^{\prime} \leq z_{d}-k} \\
& =\left(\left.\left(\left(A^{-1}\right)^{t}\right)_{t, r}\right|_{1 \leq t \leq z_{d}-k}\right) \cdot\left(\left.\left(\left(A^{-1}\right)^{t}\right)_{t^{\prime}, r^{\prime}}^{t}\right|_{1 \leq t^{\prime} \leq z_{d}-k}\right)^{t} \\
& =S \cdot S^{t} .
\end{aligned}
$$

By lemma 4.1;

$$
\operatorname{det}\left(R \cdot R^{t}\right)=\operatorname{det}(A)^{2} \cdot \operatorname{det}\left(S \cdot S^{t}\right) .
$$

Hence, one sees that, in the notation of theorem 2.1 .

$$
\begin{aligned}
& \operatorname{det}\left(j_{W}^{\star} \cdot j_{W}\right)=\operatorname{det}\left(R \cdot R^{t}\right)=(\operatorname{det}(A))^{2} \cdot \operatorname{det}\left(S \cdot S^{t}\right) \\
& =\left(\frac{t_{d-1}\left(X_{V}\right)}{t_{d-1}(X)}\right)^{2} \cdot\left(\frac{t_{d-1}\left(X_{V}\right)}{t_{d-1}\left(X_{W}\right)}\right)^{-2} \cdot \operatorname{det}\left(B[W]^{t} B[W]\right)=\left(\frac{t_{d-1}\left(X_{W}\right)}{t_{d-1}(X)}\right)^{2} \cdot \operatorname{det}\left(B[W]^{t} B[W]\right)
\end{aligned}
$$

As noted above, $B[W]$ is an integral valued matrix and $\frac{t_{d-1}\left(X_{W}\right)}{t_{d-1}(X)}$ is an integer when $W$ is a k augmented spanning forest. So this completes the proof of theorem 2.1 .

\section{Proof of lemma 4.1}

One first notes that by the Cauchy-Binet theorem the determinant of $R \cdot R^{t}$ equals the sum of squares of the $r \times r$ minors of $A$ which occur in the first $r$ rows. Similarly, the determinant of $S \cdot S^{t}$ equals the sum of squares of the $s \times s$ minors of $\left(A^{-1}\right)^{t}$ which occur in the last $s$ rows. Hence, the above lemma will follow from the following lemma:

Lemma 4.2. Let $A$ be a square $(r+s) \times(r+s)$ non-singular matrix, i.e, $\operatorname{det}(A) \neq 0$. Let $E$ be a $r \times r$ minor of $A$ and $F$ be the complementary $s \times s$ minor of $\left(A^{t}\right)^{-1}$, then

$$
\operatorname{det}(E)= \pm \operatorname{det}(A) \cdot \operatorname{det}(F) \text {. }
$$

Now replacing $A$ by pre and post multiplication by permutation matrices, with care about signs, this last lemma is reduced to:

Lemma 4.3. Let $A$ be a square $(r+s) \times(r+s)$ non-singular matrix, i.e, $\operatorname{det}(A) \neq 0$. Let $E$ be the top left $r \times r$ block of $A$ and $F$ be the bottom $s \times s$ block of $\left(A^{t}\right)^{-1}$. Then

$$
\operatorname{det}(E)=\operatorname{det}(A) \cdot \operatorname{det}(F) \text {. }
$$

By continuity, it suffices to consider the case with $\operatorname{det}(E) \neq 0, \operatorname{det}(F) \neq 0$. For these cases this lemma follows from the standard theorem.

Theorem 4.4. Let $A$ be a square $(r+s) \times(r+s)$ non-singular matrix, of the block partitioned form:

$$
A=\left(\begin{array}{ll}
a & b \\
c & d
\end{array}\right)
$$


where $a, b, c, c$ are the $r \times r, r \times s, s \times r, s \times s$ matrices respectively, defined by $a=\left(\left.A_{i, j}\right|_{1 \leq i \leq r, 1 \leq j \leq r}\right), b=$ $\left(\left.A_{i, j}\right|_{1 \leq i \leq r, r+1 \leq j \leq r+s}\right), c=\left(\left.A_{i, j}\right|_{r+1 \leq i \leq r+s, 1 \leq j \leq r}\right), d=\left(\left.A_{i, j}\right|_{r+1 \leq i \leq r+s, r+1 \leq j \leq r+s}\right)$ respectively.

If $\operatorname{det}(a) \neq 0$, then $\operatorname{det}(A)=\operatorname{det}(a) \cdot \operatorname{det}\left(d-c a^{-1} b\right)$. In particular, the inverse $\left(d-c a^{-1} b\right)^{-1}$ exists in the context $\operatorname{det}(A) \neq 0, \operatorname{det}(a) \neq 0$.

If $\operatorname{det}(d) \neq 0$, then $\operatorname{det}(A)=\operatorname{det}(d) \cdot \operatorname{det}\left(a-b d^{-1} c\right)$ In particular, the inverse $\left(a-b d^{-1} c\right)^{-1}$ exists in the context $\operatorname{det}(A) \neq 0, \operatorname{det}(d) \neq 0$.

If $\operatorname{det}(A) \neq 0, \operatorname{det}(a) \neq 0, \operatorname{det}(d) \neq 0$, then the inverse of $A, A^{-1}$, has the block decomposition :

$$
\begin{aligned}
A^{-1}= & \left(\begin{array}{cc}
\left(a-b d^{-1} c\right)^{-1} & -\left(a-b d^{-1} c\right)^{-1} b d^{-1} \\
-\left(d-c a^{-1} b\right)^{-1} c a^{-1} & \left(d-c a^{-1} b\right)^{-1}
\end{array}\right) \\
& =\left(\begin{array}{cc}
\left(a-b d^{-1} c\right)^{-1} & -a^{-1} b\left(d-c a^{-1} b\right)^{-1} \\
-d^{-1} c\left(a-b d^{-1} c\right)^{-1} & \left(d-c a^{-1} b\right)^{-1}
\end{array}\right)
\end{aligned}
$$

The proof of this is elementary. By $\operatorname{det}(a) \neq 0$, one may reduce $A$ to Schure complementary form by multiplying by a matrix of determinant 1 ; as in:

$$
\left(\begin{array}{cc}
a & 0 \\
c & d-c a^{-1} b
\end{array}\right)=\left(\begin{array}{cc}
a & b \\
c & d
\end{array}\right) \cdot\left(\begin{array}{cc}
I d & -a^{-1} b \\
0 & I d
\end{array}\right)
$$

Hence, $\operatorname{det}(A)=\operatorname{det}(a) \cdot \operatorname{det}\left(d-c a^{-1} c b\right)$ as claimed. Similarly for the case $\operatorname{det}(d) \neq 0$.

The formulas for $A^{-1}$ follow by direct calculation of $A^{-1} \cdot A$.

\section{The mesh matrix for $d$ boundaries; evaluation of its characteristic polynomial}

There is a parallel development for the mesh matrix on $d$ boundaries.

One fixes a choice of basis for the integral d-dimensional boundaries $B_{d}(X ; Z)$ say $\left\{b_{r}\right\}$ and declares that they form an orthonormal basis for the boundaries.

Let $V \subset S_{d}$ be called a spanning coforest if the restriction mapping

$$
k_{V}: B_{d}(X ; Q) \subset C_{d}(X ; Q) \stackrel{\text { restrict }}{\rightarrow} C_{d}\left(X_{V} ; Q\right)
$$

is an isomorphism. In particular, $|V|=b_{d}=\operatorname{rank} B_{d}(X ; Q)$. This is called by Lyons [18] a cobase.

By incrementally making appropriate choices of $d$-cells in $S_{d}$, the image of the restriction can be made larger and larger until it achieves the maximal value $b_{d}$. Hence, such spanning coforests always exist.

Now let $W$ be the result of deleting $k$ cells [ with $0 \leq k<b_{d}$ ], from a spanning coforest, say $V$. The result is called a $\mathbf{k}$ reduced spanning coforest. It is characterized by $|W|=b_{d}-k$ and

$$
k_{W}: B_{d}(X ; Q) \subset C_{d}(X ; Q) \stackrel{\text { restrict }}{\rightarrow} C_{d}\left(X_{W} ; Q\right)
$$

is surjective. Naturally, a spanning coforest is just a 0 reduced spanning coforest in this terminology.

This $k_{W}$ is obtained by tensoring with the rationals the composite mapping

$$
k_{W}: B_{d}(X ; Z) \subset C_{d}(X ; Z) \rightarrow C_{d}\left(X, X_{W^{c}} ; Z\right)=C_{d}\left(X_{W} ; Z\right),
$$


which is rationally a surjection by $W$ a k reduced spanning coforest. Its adjoint with respect to the orthonormal basis $\left\{b_{r}\right\}$ of $B_{r}(X ; Q)$ and the cellular basis [declared to be orthonormal] of $C_{d}\left(X_{W^{c}} ; Z\right)$ is the inclusion :

$$
k_{W}^{\star}: C_{d}\left(X_{W} ; Z\right) \hookrightarrow B_{d}(X ; Z) .
$$

Again, one calls the determinant $\operatorname{det}\left(k_{W} \cdot k_{W}^{\star}\right)$ the covolume squared of this inclusion $k_{W}^{\star}$ of lattices.

Analogously to the mesh cycle cases, let $\left\{b_{s}\right\}$ be a fixed choice of integral basis for $B_{d}(X ; Z)$ and introduce the boundary mesh matrix

$$
M\left\{b_{s}\right\}=\left\{<b_{i}, b_{j}>\right\}
$$

where one includes $B_{d}(X ; Z) \subset C_{d}(X ; Z)$ and used the cellular basis to define the inner product on d chains. Equivalently, this boundary mesh matrix is $k_{X} \cdot k_{X}^{\star}$. with the adjoint computed with respect to the respective basis $\left\{b_{s}\right\}$ on $B_{d}(X ; Z)$ and the cellular basis for the $d$ chains made orthonormal in the respective inner products.

As seen before, the application of lemma 3.2 evaluates the $b_{d}-k$ elementary symmetric function of the eigenvalues of the boundary mesh matrix $M\left(\left\{b_{s}\right\}\right)$ as a sum :

$$
\sigma_{b_{d}-k}\left(M\left(\left\{b_{s}\right\}\right)\right)=\Sigma_{W} \text { with }|W|=b_{d}-k \operatorname{det}\left(k_{W} \cdot k_{W}^{\star}\right)
$$

Again the determinant is non-zero only for the case that $k_{W}$ is onto, that is the case that $W$ is a $\mathrm{k}$ reduced spanning coforest. Hence,

$$
\sigma_{b_{d}-k}\left(M\left(\left\{b_{s}\right\}\right)\right)=\Sigma_{W} \text { with } W \text { a k reduced spanning coforest } \operatorname{det}\left(k_{W} \cdot k_{W}^{\star}\right) .
$$

For a k-reduced spanning coforest $W$ chose a spanning coforest $V$ with $W \subset V$. That is, $W$ is obtained from $V$ by deleting $k$ oriented cells, say $I$, so $V=W \cup I$ and $|I|=k$. Having made such a choice of $V$, consider the inclusion mapping :

$$
x_{W}: B_{d}\left(X_{W^{c}} ; Z\right) \subset B_{d}(X ; Z)
$$

For a choice of integral basis for $\left.B_{d}\left(X_{W^{c}} ; Z\right)=B_{d}\left(X_{V^{c} \cup I} ; Z\right)\right)$ and the chosen basis $\left\{b_{s}\right\}$ for $B_{d}(X ; Z)$ let $B^{\prime}[W]$ denote the associated integral matrix. Hence, the $b_{d} \times b_{d}$ matrix $B^{\prime}[W]^{t} B^{\prime}[W]$ represents $x_{W}^{\star} \cdot x_{W}^{\star}$ and $\operatorname{det}\left(B^{\prime}[W]^{t} B^{\prime}[W]\right)$ is independent of the choice of integral basis of $\left.B_{d}\left(X_{W^{c}} Z\right)=B_{d}\left(X_{V^{c} \cup I} ; Z\right)\right)$.

By $W$ a k reduced spanning coforest equal to $W=V-I,|I|=k$ for a spanning coforest $V$, the restriction mapping

$$
y_{W}: B_{d}\left(X_{W^{c}} ; Z\right)=B_{d}\left(X_{V^{c} \cup I} ; Z\right) \mapsto C_{d}\left(X_{I} ; Z\right)
$$

will be a rational isomorphism.

The parallel result to theorem 2.1 for the mesh matrix for boundaries $M\left\{b_{s}\right\}$ is:

Theorem 5.1. For $V$ a d dimensional spanning coforest of $X$, the group $H_{d}\left(X ; X_{V^{c}} ; Z\right)$ is a torsion subgroup. Let its order be denoted by $v(V, X)$. 
The constant coefficient of $(-1)^{b_{d}} \operatorname{det}\left(t I d-M\left\{b_{r}\right\}\right)$ is

$$
\begin{aligned}
& \operatorname{det}\left(M\left\{b_{s}\right\}\right)=\text { product of eigenvalues of } M\left\{b_{s}\right\}=\sigma_{b_{d}}\left(M\left\{b_{s}\right\}\right) \\
& =\Sigma_{V} \text {, a spanning coforest of dimension d }(v(V, X))^{2} .
\end{aligned}
$$

Also, for $V$, any spanning coforest, i.e, a 0 reduced spanning coforest,

covolume squared of $\left(k_{V}^{\star}: C_{d}\left(X_{V} ; Z\right) \hookrightarrow B_{d}(X ; Z)\right)=\operatorname{det}\left(k_{V} \cdot k_{V}^{\star}\right)=(v(V, X))^{2}$.

Quite generally, let $W$ be a d-dimensional $k$ reduced spanning coforest in $X$. For such $W$, the quotient $C_{d}\left(X_{W^{c}} ; Z\right) / x_{W}\left(Z_{d}(X ; Z)\right)$ is a torsion group isomorphic to $H_{d}\left(X ; X_{W^{c}} ; Z\right)$. Let the order of this finite torsion group be denoted by $v(V, X)$.

Moreover for $W$ contained in a spanning coforest $V$ with $W=V-I$ for $|I|=k$, then the quotient $\left.C_{d}\left(X_{I} ; Z\right) / y_{W}\left(B_{d}\left(X_{W^{c}}\right) ; Z\right)\right)$ is a torsion group and is isomorphic to $H_{d}\left(X_{W^{c}}, X_{V^{c}} ; Z\right)$. Let the order of this finite torsion group be denoted by $u(W, V)$.

It is claimed that the quotient

$$
f(W)=\frac{u(W, V)}{v(V, X)}
$$

is independent of the choice of $V$ and moreover $f(W) v(W, X)$ is an integer.

If $0 \leq k<b_{d}$, then

coefficient of $(-1)^{b_{d}-k} t^{k}$ in $\operatorname{det}\left(t I d-M\left\{b_{r}\right\}\right)$

$=\left(b_{d}-k\right)^{t h}$ elementary symmetric function in eigenvalues of $M\left\{b_{r}\right\}=\sigma_{b_{d}-k}\left(M\left\{b_{r}\right\}\right)$

$=\Sigma_{W, \text { a k reduced spanning coforest }}\left(\frac{u(W, V)}{v(V, X)}\right)^{2} \operatorname{det}\left(\left(B^{\prime}[W]\right)^{t} B^{\prime}[W]\right)$.

Moreover, for $W$, a $k$ augmented spanning forest, with $0 \geq k<z_{d}$ :

$$
\begin{aligned}
& \text { covolume squared of }\left(k_{W}^{\star}: C_{d}\left(X_{W} ; Z\right) \hookrightarrow B_{d}(X ; Z)\right) \\
& =\left(\frac{u(W, V)}{v(V, X)}\right)^{2} \operatorname{det}\left(\left(B^{\prime}[W]\right)^{t} B^{\prime}[W]\right)
\end{aligned}
$$

In particular, the coefficients of the above sum over $k$ reduced spanning coforest yielding $\sigma_{b_{d}-k}\left(M\left\{b_{r}\right\}\right)$ are all integers.

Note that if $H_{d}(X ; Q)=0$, then $Z_{d}(X ; Q)=B_{d}(X ; Q)$ and each spanning forest $W$ has complement exactly a spanning coforest $W^{c}$ and the complement of a k augmented spanning fores is a k-reduced spanning coforest. In particular, in this situation theorems 2.1] and 2.1 become identical.

\section{Proof of the main theorem for mesh matrix for $d$ boundaries: Theorem 5.1}

Now consider the case $V$ a spanning coforest, i.e., $k=0$, the mappings $k_{V}, k_{V}^{\star}$ are rationally isomorphisms. Hence, the determinant $\operatorname{det}\left(k_{V} \cdot k_{V}^{\star}\right)$ is the square of the order of the finite abelian group:

$$
C_{d}\left(X_{V} ; Z\right) /\left\{\text { image by } k_{V} \text { of } B_{d}(X ; Z)\right\}
$$

It is claimed that this finite torsion group has order $v(V, X)$ completing the proof for the case $k=0$.

Quite generally, let $W$ be a k reduced spanning forest, then $k_{W}: B_{d}(X ; Z) \rightarrow C_{d}\left(X_{V} ; Z\right)$ is rationally onto, so the quotient $C_{d}\left(X_{W} ; Z\right) /\left\{\right.$ image by $k_{W}$ of $\left.B_{d}(X ; Z)\right\}$ is a torsion 
ENUMERATIVE COMBINATORICS OF SIMPLICIAL AND CELL COMPLEXES: KIRCHHOFF AND TRENT TYPE THEOREMS group. It is claimed that the order of this finite quotient group

$$
\mid C_{d}\left(X_{W} ; Z\right) /\left\{\text { image by } k_{W} \text { of } B_{d}(X ; Z)\right\} \mid
$$

is $v(W, X)$.

The mapping $k_{W}$ may be reinterpreted [ since $X_{W}, X_{W^{c}}$ each have the same $d-1$ skeleton as $X$ :

$$
k_{W}: B_{d}(X ; Z) \stackrel{\text { onto }}{\rightarrow} B_{d}\left(X, X_{W^{c}} ; Z\right) \subset Z_{d}\left(X, X_{W^{c}} ; Z\right)=C_{d}\left(X_{W} ; Z\right)
$$

So this finite abelian quotient is identified with $H_{d}\left(X, X_{W^{c}} ; Z\right)$. Its order is $v(W, X)$.

Finally, in view of the long exact sequence of the triple $\left(X, X_{W^{c}}, X_{V^{c}}\right)$, there is an exact sequence

$$
H_{d}\left(X_{W^{c}}, X_{V^{c}} ; Z\right) \rightarrow H_{d}\left(X, X_{V^{c}} ; Z\right) \rightarrow H_{d}\left(X, X_{W^{c}} ; Z\right) \rightarrow H_{d-1}\left(X_{W^{c}}, X_{V^{c}} ; Z\right)=0
$$

since $X_{W^{c}}, X_{V^{c}}$ have the same (d-1) skeleton. By the above all these are finite torsion groups. Hence, by exactness, $u(W, V) /(v(V, X) / v(W, X))=\frac{u(W, V)}{v(V, X)} \cdot v(W, X)$ is an integer as desired.

Next consider again as above $V$ a spanning coforest with $W=V-I$ for $|I|=k$ oriented cells of $V$. That is, $W$ is a k reduced spanning coforest. Now the mapping

$$
y_{W}: B_{d}\left(X_{W^{c}} ; Z\right)=B_{d}\left(X_{V^{c} \cup I} ; Z\right) \rightarrow C_{d}\left(X_{I} ; Z\right)=C_{d}\left(X_{V^{c} \cup I}, X_{V^{c}} ; Z\right)
$$

is rationally an isomorphism. Recall that $W^{c}=V^{c} \cup I$. It can be factored as

$$
B_{d}\left(X_{W^{c}} ; Z\right) \stackrel{\text { onto }}{\rightarrow} B_{d}\left(X_{W^{c}}, X_{V^{c}} ; Z\right) \subset Z_{d}\left(X_{W^{c}}, X_{V^{c}} ; Z\right)=C_{d}\left(X_{W^{c}}, X_{V^{c}} ; Z\right) .
$$

Hence, the quotient $C_{d}\left(X_{I} ; Z\right) / y_{W}\left(B_{d}\left(X_{W^{c}} ; Z\right)\right)$ is a finite torsion group and is isomorphic to $H_{d}\left(X_{W^{c}}, X_{V^{c}} ; Z\right)$. Its order is $u(W, V)$.

Let $A^{\prime}$ be the $n_{d} \times b_{d}$ matrix represent the mapping $k_{X}: B_{d}(X ; Z) \rightarrow C_{d}(X ; Z)$ with respect to the chosen basis $\left\{b_{s}\right\}$ for $B_{d}(X ; Z)$ and the cellular basis for $C_{d}(X ; Z)$. In these terms the boundary mesh matrix, $M\left(\left\{b_{s}\right\}\right)$, is just $\left(A^{\prime}\right)^{t} \cdot\left(A^{\prime}\right)$.

Now to evaluate the covolume $\operatorname{det}\left(k_{W} \cdot k_{W}^{\star}\right)$ for $W$ a $\mathrm{k}$ reduced spanning coforest with $0<k<b_{d}$, pick a spanning coforest $V$ of $X$ with $W \subset V$. Let $W=V-I$ with $|I|=k$.

Then by $V$ a spanning coforest the restriction mapping

$$
k_{V}: B_{d}(X ; Z) \rightarrow C_{d}\left(X_{V} ; Z\right)
$$

is rationally an isomorphism. Also by above, the quotient is of finite order $v(V, X)$.

In particular, $k_{V}$ is rationally an isomorphism. so for each $\sigma_{j} \in V$ [oriented as in $S_{d}$ ] there is a unique $\mathrm{d}$ boundary $b\left(\sigma_{j}\right) \in B_{d}(X ; Q)$ which restricts to $1 \cdot \sigma$ in $C_{d}\left(X_{W} ; Q\right)$. That is, $b\left(\sigma_{j}\right)$ lies in $B_{d}\left(X_{V^{c} \cup \sigma_{j}} ; Q\right)$ Here $\sigma_{j}$ ranges over $V$ which is of size $b_{d}$, so $1 \leq j \leq b_{d}$.

Under the identification above of $B_{d}(X ; Q)$ with $C_{d}\left(X_{V}\right)$, identifying $b\left(\sigma_{j}\right)$ with $1 \cdot \sigma_{j}$ for $\sigma_{j} \in V$, the map $k_{V}$ is identified with the identity mapping

$$
I d: B_{d}(X ; Q) \rightarrow B_{d}(X ; Q)
$$

with $A^{\prime}$ recording the change of basis mapping

$$
b_{s}=\Sigma_{\sigma_{j} \in V, j=1, \cdots b_{d}} A_{\sigma_{j}, s}^{\prime} b\left(\sigma_{j}\right) .
$$


Note that there ia a direct sum decomposition

$$
\left.B_{d}(X ; Q)=B_{d}\left(X_{V^{c} \cup W}, Q\right)\right) \oplus\left(B_{d}(X ; Q) \cap C_{d}\left(X_{V^{c} \cap I} ; Q\right)\right.
$$

which is compatible with the orthonormal basis given by the geometric generators $\left\{b\left(\sigma_{j}\right) \mid \sigma_{j} \in\right.$ $V\}$. The first summand has basis $\left\{b\left(\sigma_{j}\right) j=1 \cdots b_{d}-k\right\}=\left\{b\left(\sigma_{j}\right) \mid \sigma_{j} \in W\right\}$; the second summand has basis $\left\{b\left(\sigma_{j}\right) \mid \sigma_{j} \in I\right\}$.

Arrange that the first $b_{d}-k$ cells of $V$ be those of $W=V-I$, and let $R^{\prime}$ be the matrix obtained from $A^{\prime}$ by deleting the last $k$ rows. Then $R^{\prime}$ represents $k_{W}$ and $k_{W} \cdot k_{W}^{\star}$ is represented by $R^{\prime}\left(R^{\prime}\right)^{t}$.

As before let $S$ be the last $k$ rows of $\left(\left(A^{\prime}\right)^{-1}\right)^{t}$. Then $S^{t}$ represents the mapping

$$
B_{d}\left(X_{W^{c}} ; Z\right)=B_{d}\left(X_{V^{c} \cap I} ; Q\right) \subset B_{d}(X ; Q)
$$

with the geometric basis used as a basis for the domain and the the fixed chosen basis $\left\{b_{s}\right\}$ for the range.

Converting from the geometric basis on the domain to any choice of integral basis for $B_{d}\left(X_{W^{c}}, ; Z\right)$ entails a change with determinant the same as the order of

$$
C_{d}\left(X_{I} ; Z\right) / y_{W}\left(B_{d}\left(X_{W^{c}} ; Z\right)\right)
$$

which is by definition $u(W, V)$. That is,

$$
\operatorname{det}\left(S S^{t}\right)=u(W, V)^{2} \operatorname{det}\left(B[W]^{\prime}\left(B[W]^{\prime}\right)^{t}\right)
$$

Appealing to lemma 4.1 gives the desired result

$$
\begin{aligned}
& \operatorname{det}\left(k_{W} \cdot k_{W}^{\star}\right)=\operatorname{det}\left(R^{\prime} \cdot\left(R^{\prime}\right)^{t}\right) \\
& =\operatorname{det}\left(A^{\prime}\right)^{2} \operatorname{det}\left(\left(S^{\prime}\right)\left(S^{\prime}\right)^{t}\right)=\operatorname{det}\left(A^{\prime}\right)^{2} u(W, V)^{-2} \operatorname{det}\left(B^{\prime}[W]\left(B[W]^{\prime}\right)^{t}\right) \\
& =(u(W, V) / v(V, X))^{2} \operatorname{det}\left(B^{\prime}[W]\left(B[W]^{\prime}\right)^{t}\right)
\end{aligned}
$$

as desired.

Since the determinant $\operatorname{det}\left(k_{W} \cdot k_{W}^{\star}\right)$ as a covolume of an integral lattice in the standard lattice with standard inner product is an integer, the quantity $(u(W, V) / v(V, X))^{2} \operatorname{det}\left(B^{\prime}[W]\left(B[W]^{\prime}\right)^{t}\right)$ is an integer also.

The equality $\operatorname{det}\left(k_{W} \cdot k_{W}^{\star}\right)=(u(W, V) / v(V, X))^{2} \operatorname{det}\left(B^{\prime}[W]\left(B[W]^{\prime}\right)^{t}\right)$ proves that the quotient $f(W)=\frac{u(W, V)}{v(V, X)}$ is independent of the choice of $V$.

\section{Evaluation of all coefficients of mesh matrices for cycles and boundaries with respect to geometrically defined bases.}

Let $V_{0} \subset S_{d}$ be a fixed choice of d dimensional spanning forest of $X$. As explained in $§ 2$, there is a natural geometric basis for the rational $d$-cycles $Z_{d}(X ; Q)$ described as follows: For each oriented d cell, say $\sigma_{j}$ in $S_{d}-V_{0}$, let $z\left(\sigma_{j}\right)$ be the unique d cycle in $Z_{d}\left(F \cup \sigma_{j} ; Q\right)$ which evaluates to +1 on $\sigma_{j}$. As explained there, as $\sigma_{j}$ ranges over $S_{d}-V_{0}$ the d cycles $z\left(\sigma_{j}\right)$ give a basis for the rational vector space $Z_{d}(X ; Q)$ of d cycles on $X$. Also, for any subset $U \subset S_{d}-V_{0}$, the elements $\left\{z\left(\sigma_{j}\right) \mid \sigma_{j} \in U\right\}$ forms a rational basis for $Z_{d}\left(V_{0} \cup U ; Q\right)$.

Associated to this geometric basis $\left\{z\left(\sigma_{j}\right)\right\}$ one defines the cycle mesh matrix of $X$ for this geometric basis using $V_{0}$ as $M_{\text {cycle },\left(V_{0}, X\right)}\left\{z\left(\sigma_{j}\right)\right\}$ via $\left(\sigma, \sigma^{\prime}\right) \mapsto<z(\sigma), z\left(\sigma^{\prime}\right)>_{X}$ with the pairing defined using the cell complex $X$. 
ENUMERATIVE COMBINATORICS OF SIMPLICIAL AND CELL COMPLEXES: KIRCHHOFF AND TRENT TYPE THEOREN\&\$

Theorem 7.1. Let $V_{0}$ be a d dimensional spanning forest, then the cycle mesh matrix $M_{\text {cycle },(L, X)}\left\{z\left(\sigma_{j}\right)\right\}$ is a $z_{d} \times z_{d}$ matrix with coefficient of $(-1)^{k} t^{z_{d}-k}$ the $k^{\text {th }}$ elementary symmetric function of its eigenvalues, called here $\sigma_{k}\left(V_{0}\right)$. Moreover, there is a direct combinatorial formula for this coefficient:

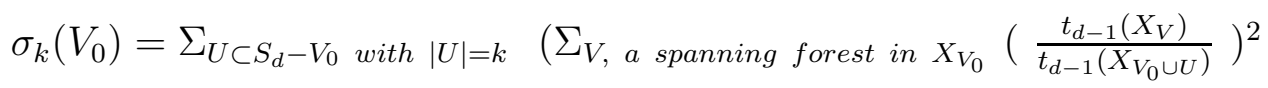

Analogously, let $V_{1} \subset S_{d+1}$ be a fixed choice of $d+1$ dimensional spanning forest of $X$. Again, there is a natural geometric basis for the $d$ boundaries $B_{d}(X ; Q)$. It consists of the boundaries $\left\{\partial_{d+1}(1 \cdot u) \mid u \in S_{d+1}-V_{1}\right\}$. Also, for each subcomplex of the form $X^{(d)} \cup V_{1} \cup U$ with $U \subset S_{d+1}-V_{1}$, the images $\left\{\partial_{d+1}(1 \cdot u) \mid u \in S_{d+1}-V_{1}\right.$ and $\left.u \in U\right\}$ form a rational basis for $B_{d}\left(X^{(d)} \cup V_{1} \cup U ; Q\right)$, the boundaries.

Associated to this geometric basis $\left\{\partial_{d+1}(u) \mid u \in S_{d+1}-V_{1}\right\}$, one defines the boundary mesh matrix of $X$ for this geometric basis defined by $V_{1}$ as

$M_{\text {boundary },\left(V_{1}, X\right)}\left(\left\{\partial_{d+1}(u) \mid u \in S_{d+1}-V_{1}\right\}\right)$ via $(u, v) \mapsto<\partial_{d+1}(u), \partial_{d+1}(v)>_{X}$ with the pairing defined using the cell complex $X$.

Theorem 7.2. Let $V_{1}$ be a $d+1$ spanning forest, then the boundary mesh matrix $M_{\text {boundary },\left(V_{1}, X\right)}\left(\left\{\partial_{d+1}(u) \mid u \in S_{d+1}-V_{1}\right\}\right)$ is a $b_{d} \times b_{d}$ matrix with coefficient of $(-1)^{k} t^{b_{d}-k}$ the $k^{\text {th }}$ elementary symmetric function of its eigenvalues, called here $\sigma_{k}\left(V_{1}\right)$. Moreover, there is a direct combinatorial formula for this coefficient:

$\sigma_{k}\left(V_{1}\right)=\Sigma_{U \subset V_{1} \text { with }|U|=k} \quad\left(\Sigma_{V, \text { a d dimensional spanning coforest in } X^{(d)} \cup V_{1} \cup U}\left|H_{d-1}\left(X_{V_{1}}, X_{W^{c}} ; Z\right)\right|^{2}\right)$ where $\left|H_{d-1}\left(X_{V_{1}}, X_{W^{c}} ; Z\right)\right|$ is the order of the finite torsion group $H_{d-1}\left(X_{V_{1}}, X_{W^{c}} ; Z\right)$.

with $X_{V_{1}}=X^{(d)} \cup V_{1}$

and $X_{W^{c}}=X^{(d-1)} \cup\left(S_{d}-W\right)$. This weight is alternately expressed as

the integer $\left[\frac{t_{d-1}\left(X_{W}\right) t_{d-2}\left(X_{\left(V_{1}\right)}\right) t_{d-1}^{\prime}\left(V_{1}\right)}{t_{d-2}(X)}\right]$, see the next section.

The proofs of these two theorems proceeds as follows:

For theorem 7.1, applying lemmas 3.1, 3.2, one sees that $\sigma_{k}\left(V_{0}\right)$ is expressed as a double sum over $U \subset S_{d}-V_{0}$ with $|U|=k$ and over $Y \subset S_{d}$ of size $k$ of a square of the determinant for the restriction mapping $Z_{d}\left(V_{0} \cup U ; Q\right)$ to $C_{d}(Y ; Q)$. This last mapping is onto [and hence an isomorphism] only if $Y \subset\left(V_{1} \cup U\right)$ and $V=\left(V_{1}-Y\right)$ is a spanning forest for $X_{V_{1} \cup U}$. In this instance, the matrix at issue is that for changing from the geometrical basis for the spanning forest $V_{0}$ in $X_{V_{0} \cup U}$ to the geometrical basis for the spanning forest $V$ of the same cell complex $X_{V_{0} \cup U}$. These two bases can be compared to the integral basis of $Z_{d}\left(X_{V_{0} \cup U} ; Z\right)$. by invoking theorem 2.1. On that basis, the desired determinant is $\left(\frac{t_{d-1}\left(X_{V_{0}}\right)}{t_{d-1}\left(X_{V_{0} \cup U}\right)}\right)^{-1}\left(\frac{t_{d-1}\left(X_{V}\right)}{t_{d-1}\left(X_{V_{0} \cup U}\right)}\right)=$ $\frac{t_{d-1}\left(X_{V}\right)}{t_{d-1}\left(X_{V_{0}}\right)}$.

For theorem 7.2, applying lemmas 3.1, 3.2, one sees that $\sigma_{k}\left(V_{1}\right)$ is expressed as a double sum over $U \subset V_{1}$ with $|U|=k$ and over $V \subset S_{d}$ of size $k$ of a square of the determinant for the mapping

$$
C_{d+1}\left(X^{(d)} \cup V_{1}, Z\right) \stackrel{\partial_{d+1}}{\rightarrow} B_{d}\left(X^{(d)} \cup V, Z\right) \subset C_{d}(X ; Z) \stackrel{\text { restriction }}{\rightarrow} C_{d}\left(X^{(d)} \cup V, Z\right) .
$$

This last mapping is onto [and hence an isomorphism] only if $V \subset X^{(d)} \cup U$ and $V$ is a d dimensional spanning coforest for $X^{(d)} \cup U$. As the next section proves, in this case the mapping is rationally an isomorphism with quotient group a finite order equal to the order 
of the torsion group $H_{d-1}\left(X_{V_{1}}, X_{W^{c}} ; Z\right)$, Here $X_{V_{1}}=X^{(d)} \cup V_{1}$ and $X_{W^{c}}=X^{(d-1)} \cup\left(S_{d}-W\right)$. This is alternately expressed there as the integer $\left[\frac{t_{d-1}\left(X_{W}\right) t_{d-2}\left(X_{\left(V_{1}\right)}\right) t_{d-1}^{\prime}\left(V_{1}\right)}{t_{d-2}(X)}\right]$.

\section{A Kirchhoff type theorem for cell complexes, extending Lyons's [18] work to the complete characteristic polynomial}

In the same manner:

Let $A$ be the integer valued matrix representing the boundary mapping with respect to the cellular basis

$$
\partial_{d}: C_{d}(X ; Z) \rightarrow C_{d-1}(X ; R) .
$$

Then $A^{t}$ represents the adjoint for the cellular inner product $<.,>$. Recall that $\partial_{d}$ has rank $b_{d-1}=\operatorname{dim} B_{d-1}(X ; R)$

Let $0<m \leq b_{d-1}$ with $S_{d}$ the $d$ cells with their specified orientations.

By the same algebra as before, one gets

$$
\begin{aligned}
& m^{\text {th }} \text { elementary symmetric function of the eigenvalues of } \partial \cdot \partial^{*} \\
& =\Sigma_{I \subset S_{d-1}} \quad J \subset S_{d} \text { with }|I|=|J|=m\left[\operatorname{det}\left(A_{(I, J)}\right]^{2}\right. \text {. }
\end{aligned}
$$

For the term to be non-zero, the mapping $A_{(I, J)}$ must be an isomorphism after tensoring with the reals.

The matrix $A_{(I, J)}$ represents the composite :

$$
C_{d}\left(X_{J} ; Z\right) \subset C_{d}(X ; Z) \stackrel{\partial}{\rightarrow} B_{d-1}(X ; Z) \subset C_{d-1}(X ; Z) \stackrel{\text { restrict }}{\rightarrow} C_{d-1}\left(X_{I} ; Z\right)
$$

For $\operatorname{det}\left(A_{(I, J)}\right) \neq 0$, necessarily the composite

$$
C_{d}\left(X_{J} ; R\right) \subset C_{d}(X ; R) \stackrel{\partial}{\rightarrow} B_{d-1}(X ; R)
$$

must be one to one. That is, $Z_{d}\left(X_{J} ; Z\right)=0$.

The conventional notation for this is : $J \subset S_{d}$ is called a forest of size $|J|=m$. That is, $X_{J}$ carries no $d$-cycles.

Additionally, for $\operatorname{det}\left(A_{(I, J)}\right) \neq 0$, necessarily the composite

$$
B_{d-1}\left(X_{J} ; R\right) \subset B_{d-1}(X ; R) \subset C_{d-1}(X ; R) \stackrel{\text { restrict }}{\rightarrow} C_{d-1}\left(X_{I} ; R\right)
$$

must be an isomorphism. That is, $I \subset S_{d-1}$ interpreted via $X_{I}=X^{d-2} \cup I$ inside $X_{J}=$ $X^{d-1} \cup J$ must be a spanning coforest of $X_{J}$. In combination this implies that $|I|=m$ also.

Hence, the above sum is restricted to $J=V$ a (d-dimensional) forest of size $m$ and $I=W$ a ((d-1)-dimensional) spanning coforest of $X_{J}=X_{V}$. This ensures that $\operatorname{det}\left(A_{(I, J)}\right) \neq 0$.

$m^{\text {th }}$ elementary symmetric function of the eigenvalue of $\partial \cdot \partial^{\star}$

$=\Sigma_{V \subset S_{d}} W \subset S_{d-1}$ with $|V|=m,|W|=m, V$ a forest of size $m$ and $W$ a spanning coforest of $X_{V}\left[\operatorname{det}\left(A_{(W, V)}\right)\right]^{2}$

Consider the inclusion $X_{W}=X^{d-2} \cup W \subset X_{V}=X^{d-1} \cup V$, defining the mapping $A_{(W, V)}$

$$
C_{d}\left(X_{V} ; Z\right) \subset C_{d}(X ; Z) \stackrel{\partial}{\rightarrow} B_{d-1}(X ; Z) \subset C_{d-1}(X ; Z) \stackrel{\text { restrict }}{\rightarrow} C_{d-1}\left(X_{W} ; Z\right)
$$

which in the cases of interest is a rational isomorphism. 
ENUMERATIVE COMBINATORICS OF SIMPLICIAL AND CELL COMPLEXES: KIRCHHOFF AND TRENT TYPE THEORENমS

Now as A. Duval, C. Klivans, and J. Martin note [12], one can reinterpret this map more geometrically. On integral chains,

$$
C_{d}\left(X_{V}, X_{W^{c}} ; Z\right)=C_{d}\left(X_{V} ; Z\right) \stackrel{\partial}{\rightarrow} C_{d-1}\left(X_{V}\right) \stackrel{\text { restrict }}{\rightarrow} C_{d-1}\left(X_{W} ; Z\right)=C_{d-1}\left(X_{V}, X_{W^{c}} ; Z\right)
$$

becomes identified with the boundary mapping for the pair $\left(X_{V}, X_{W^{c}}\right)$. Since $C_{d-1}\left(\left(X_{V}, X_{W^{c}} ; Z\right)=\right.$ 0 and $C_{d+1}\left(\left(X_{V}, X_{W^{c}} ; Z\right)=0\right.$ and this boundary mapping is a rational isomorphism, it follows that

$$
\operatorname{det}\left(A_{(V, W)}\right)^{2}=\left|H_{d-1}\left(X_{V}, X_{W^{c}} ; Z\right)\right|^{2}
$$

in the cases of interest.

In the maximal case $k=b_{d}$, by $V$ a forest of size $b_{d}$, necessarily the mapping $C_{d}\left(X_{V} ; Z\right) \subset$

$C_{d}(X ; Z) \stackrel{\partial}{\rightarrow} B_{d-1}(X ; Z)$ is a rational isomorphism. Hence, the set of $W$ for which $W$ is a spanning coforest of $X_{V}$ is the same as the spanning coforests of $X$.

Lyons proved in the case $k=b_{d}$, that

$$
\left|H_{d-1}\left(X_{V}, X_{W^{c}} ; Z\right)\right|^{2}=\left[\frac{t_{d-1}\left(X_{W}\right) t_{d-2}\left(X_{V^{c}}\right) t_{d-1}^{\prime}(V)}{t_{d-2}(X)}\right]^{2} \in Z
$$

where for a CW complex $K$ the order of the torsion of $H_{k}(K ; Z)$ is denoted by $t_{k}(K)$ and $t_{d-1}^{\prime}(V)$ is the order of the quotient of $Z_{d-1}(X ; Z)$ by $Z_{d-1}(X) \cap B_{d-1}(X ; Q)+Z_{d}\left(X_{V^{c}} ; Z\right)$. Here by convention $X_{W}=X^{(d-1)} \cup W$ and $X_{V^{c}}=X^{(d)} \cup V^{c}$.

For $m=b_{d}$ the corresponding sum decouples, yielding the first part of the next theorem, which appears in the work of Lyons:

Theorem 8.1. $\quad$ a. Lyons [18]. The product of the non-zero eigenvalues of $\partial \cdot \partial^{\star}$, the combinatorial Laplacian, or Kirchhoff mapping, acting on $(d-1)$ chains $C_{d-1}(X ; Z) \rightarrow$ $C_{d-1}(X ; Z)$, i.e., equals

$$
=\left(\Sigma_{W, \text { forest of size } m=b_{d}}\left(t_{d-1}\left(X_{W}\right)\right)^{2}\right) \cdot\left(\Sigma_{V \text {, spanning coforest in } X}\left(\frac{t_{d-2}\left(X_{V^{c}}\right) t_{d-1}^{\prime}(V)}{t_{d-2}(X)}\right)^{2}\right) \text {, }
$$
where each term of each sum is an integer.

b. More generally, if $0<k \leq b_{d}$, the $k^{\text {th }}$ elementary symmetric function in the eigenvalues of $\partial \cdot \partial^{\star}$, the combinatorial Laplacian, or Kirchhoff map is

$$
=\Sigma_{V \text { a }} \text { forest of size } k\left[\Sigma_{W} \text { a spanning coforest of } X_{V}\left|H_{d}\left(X_{V}, X_{W^{c}} ; Z\right)\right|^{2}\right] \text {. }
$$

\section{Homology covolume squared via determinants of mesh matrices}

Naturally, the above results imply a statement about $H_{d}(X ; R)$. Recall that using the cellular basis to identify chains and cochains, the coboundary dual to $\partial_{d}: C_{d}(X ; R) \rightarrow C_{d-1}(X ; R)$ gets identified with $\partial_{d}^{\star}: C_{d-1}(X: Z) \rightarrow C_{d}(X ; Z)$. The Hodge theory decomposition becomes in this setting an orthogonal direct sum decomposition of real $d$ chains with the cellular inner product $<.,>$ :

$$
C_{d}(X ; R)=\mathcal{H}_{d} \oplus\left\{\text { image } \partial_{d+1}\right\} \oplus\left\{\text { image } \partial_{d}^{\star}\right\},
$$

with $\mathcal{H}_{d}$ the space of (d-dimensional) harmonics $\mathcal{H}_{d}=\left\{c \mid \partial_{d}(c)=0\right.$ and $\left.\partial_{d+1}^{\star}(c)=0\right\}$.

Now one has the direct sum orthogonal decomposition :

$$
Z_{d}(X ; R)=\mathcal{H}_{d} \oplus B_{d}(X ; R)
$$


Let $\overline{B_{d}(X ; Z)}=B_{d}(X ; Q) \cap Z_{d}(X ; Z)$. Then $\overline{B_{d}(X ; Z)}$ is a direct summand of $C_{d}(X ; Z)$ and the quotient $\overline{B_{d}(X ; Z)} / B_{d}(X ; Z)$ is isomorphic to the torsion in $H_{d}(X ; Z)$.

Thus the covolume squared for the lattice $B_{d}(X ; Z)$ in $C_{d}(X ; Z)$ with cellular inner product $<.,>$ is $t_{d}(X)^{2}$ times that of $\overline{B_{d}(X ; Z)}$.

Now chose a direct summand, say $G \subset Z_{d}(X ; Z)$, with

$$
Z_{d}(X ; Z)=G \oplus \overline{B_{d}(X ; Z)} .
$$

This is feasible as $\overline{B_{d}(X ; Z)}$ is a direct summand. Then the covolume of $Z_{d}(X ; Z)$ computed from this lattice is exactly the same as that of $\pi(G) \oplus \overline{B_{d}(X ; Z)}$, where $\pi_{d}$ is is the orthogonal projection of $Z_{d}(X ; Z)$ onto $\mathcal{H}_{d}$ or equivalently the direct summand $G$. This shows that

$$
\begin{aligned}
& \text { covolume squared for lattice } Z_{d}(X ; Z) \text { under }<., .> \\
& =\left(\text { covolume squared for lattice } B_{d}(X ; Z) \text { under }<., .>\right) \\
& \quad \cdot\left(\text { covolume for the lattice } \pi_{d}\left(Z_{d}(X ; Z)\right) \subset \mathcal{H}_{d} \text { under }<.,>\right) \text {. }
\end{aligned}
$$

That is, if one defines the lattice in $\mathcal{H}_{d}$ by the orthogonal projection $\pi_{d}$ of the lattice $Z_{d}(X ; Z)$ onto $\mathcal{H}_{d}$, one has

$$
\begin{aligned}
& \text { covolume squared for lattice } \pi_{d}\left(Z_{d}(X ; Z)\right) \subset \mathcal{H}_{d} \text { under }<., .> \\
& =\frac{\text { covolume squared for lattice } Z_{d}(C ; Z) \text { under }<., .>}{\text { covolume squared for lattice } B_{d}(C ; Z) \text { under }<., .>} \cdot t_{d}(X)^{2} .
\end{aligned}
$$

This gives in view of the above a combinatorial description of this "homological covolume".

\section{Combinatorial identities via a computation of Reidemeister-Franz torsion}

As explicated by Milnor [21], the R-torsion of Reidemeister-Franz [27, 13] $\tau\left(C_{\star},\left\{d_{i}\right\},\left\{e_{i, j}\right\},\left\{h_{j, k}\right\}\right)$, is an invariant which is a non-zero real number, associated to the following data:

- A finite chain complex of real finite dimensional vectors spaces, say $C_{0}, \cdots, C_{N}$ with differentials $d_{i}: C_{i} \rightarrow C_{i-1}$ with $d_{i-1} \cdot d_{i}=0$ as recorded by the sequence:

$$
0 \rightarrow C_{n} \stackrel{d_{n}}{\rightarrow} C_{n_{1}} \stackrel{d_{n-1}}{\rightarrow} \cdots \stackrel{d_{1}}{\rightarrow} C_{0} \rightarrow 0
$$

Let $C_{i}$ have real dimension $r_{i}$. Let $Z_{i}=\left\{\right.$ kernel of $\left.d_{i}: C_{i} \rightarrow C_{i-1}\right\}$ be the i-cycles; let $B_{i}=\left\{\right.$ image of $\left.d_{i+1} ; C_{i+1} \rightarrow C_{i}\right\}$ be the i-boundaries. Let $H_{i}$ be the $i^{\text {th }}$ homology, $H_{i}=Z_{i} / B_{i}$ and have rank $s_{i}$. Let $B_{i}$ have rank $t_{i}$.

- For each vector space $C_{i}$ a chosen basis, say $\left\{e_{i, j} \mid j=1, \cdots, r_{i}\right\}$

- For each homology $H_{i}$ a chosen basis, say $\left\{h_{i, k} \mid k=1, \cdots, s_{i}\right\}$

This torsion $\tau$ is defined as follows: Chose elements $\tilde{h}_{i, k} \in Z_{i} \subset C_{i}$ which project to the chosen homology classes $h_{i, k}$. Next chose elements, say $\left\{b_{i-1, l} \mid l=1, \cdots, t_{i-1}\right\}$ which are a basis for $B_{i-1}$. Next chose elements $\tilde{c}_{i, l} \in C_{i}$ with $d_{i}\left(\tilde{c}_{i, l}\right)=b_{i-1, l}$ with $l=1, \cdots, t_{i-1}$.

With these choices, $Z_{i}$ has basis $\left[\left\{\tilde{h}_{i, k}\right\} \sqcup\left\{b_{i, l}\right\}\right]$ and $C_{i}$ has the basis $X_{i}=\left[\left\{\tilde{h}_{i, k}\right\} \sqcup\left\{b_{i, l}\right\} \sqcup\left\{\tilde{c}_{i, l^{\prime}}\right\}\right]$. Now set $D_{i}=\left|\operatorname{det}\left(\left[X_{i}:\left\{e_{i, j}\right\}\right]\right)\right|$ where $\left[X_{i}:\left\{e_{i, j}\right\}\right]$ is the matrix expressing the basis $X_{i}$ in terms of the basis $\left\{e_{i, j}\right\}$. One defines the ReidemeisterFranz torsion via :

$$
\left(\tau\left(C_{\star},\left\{d_{i}\right\},\left\{e_{i, j}\right\},\left\{h_{j, k}\right\}\right)\right)^{2}=\prod_{i=0}^{N}\left(D_{i}\right)^{(-1)^{i}}
$$


A simple check shows that the R-torsion $\tau$ squared is independent of the choices of $\tilde{h}_{i, k}, b_{i-1, l}, c_{i, l^{\prime}}$ [26] ; also, the sign issues are disposed of by taking the square.

Turning to the case of a cell complex of dimension $d$, one has the cellular basis for $C_{i}(X ; R)$ given by the i-cells of $X$, once oriented. Moreover, the real homology $H_{i}(X ; R)$ has a preferred basis coming from a choice of $Z$-basis, say $\left\{h_{i, k}\right\}$ for the free part of the integral homology. Consequently, there is a canonically defined R-torsion for any finite cell complex $X, \tau\left(C_{\star}(X ; R), \partial_{R},\left\{\right.\right.$ combinatorial basis for $C_{\star}(X ; R)$ and $\left.\left.H_{\star}(X ; R)\right\}\right)$. Not surprisingly, there is a simple formula (known to experts) for it:

Theorem 10.1. The combinatorial R-torsion is given by

$$
\left[\tau\left(C_{\star}(X ; R), \partial_{R},\left\{\text { combinatorial basis for } C_{\star} \text { and } H_{\star}\right)\right\}\right]^{2}=\left[\prod_{k=1}^{d-1} \theta_{X, i}^{(-1)^{i}}\right]^{2},
$$

where $\theta_{X, i}$ is the order of the torsion in $H_{i}(X ; Z)$.

Proof: Since $Z_{i}(X ; Z)$ is a free direct summand of $C_{i}(X ; Z)$, the quotient $C_{i}(X ; Z) / B_{i}(X ; Z)$ has torsion subgroup equal to the torsion subgroup of $H_{i}(X ; Z)=$ $Z_{i}(X ; Z) / B_{i}(X ; Z)$; so in particular its order is just $\theta_{X, i}$. Alternatively expressed, let $\overline{B_{i}(X ; Z)}$ be the lattice of elements $x \in C_{i}(X ; Z)$ with $N c \in B_{i}(X ; Z)$ for an integer $N \neq 0$, then $\left|\overline{B_{i}(X ; Z)} / B_{i}(X ; Z)\right|=\theta_{X, i}$ and $\overline{B_{i}(X ; Z)}$ is a direct summand of $C_{i}(X ; Z)$.

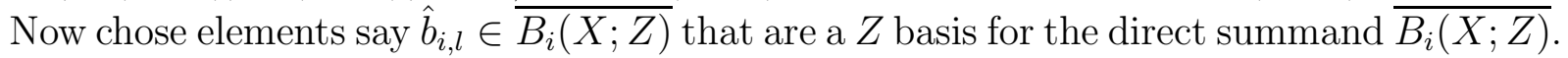
Since $Z_{i}(X ; Z) / \overline{B_{i}(X ; Z)} \cong H_{i}(X ; Z) /\{$ torsion $\}$ is free abelian, one may choose classes $\left\{\tilde{h}_{i, k}\right\}$ in $Z_{i}(X ; Z)$ which project to a $Z$-basis for $H_{i}(X ; Z) /\{$ torsion $\}$, i.e., the required combinatorial basis. With these choices made $\left\{\tilde{h}_{i, k}\right\} \sqcup\left\{\hat{b}_{i, l}\right\}$ form an integral basis for the direct summand $Z$-lattice $Z_{i}(X ; Z)$ inside $C_{i}(X ; Z)$. Again, since $C_{i}(X ; Z) / Z_{i}(X ; Z)$ is free abelian, one may chose elements $x_{i, l^{\prime}} \in C_{i}(X ; Z)$ so that they project to an integral basis of this quotient. With these choices the lattice $C_{i}(X ; Z)$ has an integral basis given by $\left\{\tilde{h}_{i, k}\right\} \sqcup\left\{\hat{b}_{i, l}\right\} \sqcup\left\{x_{i, l}\right\}$. This gives a reference basis for $C_{i}(X ; Z)$ and represents in $C_{i}(X ; R)$ a basis which differs from the cellular basis by an element in $G L\left(\operatorname{dim} C_{i}(X ; R), Z\right)$ so the transition matrix has determinant \pm 1 . Consequently, one may use this reference basis in computing the $D_{i}$ and the R-torsion $\tau$.

Naturally, one uses the chosen lift $\tilde{h}_{i, k}$ again and the classes $\left\{d_{i+1}\left(x_{i+1, l^{\prime}}\right)\right\}$ and $\left\{x_{i, l}\right\}$ in computing the R-torsion with the above reference basis. That is, $X_{i}=\left[\left\{\tilde{h}_{i, k}\right\},\left\{d_{i+1}\left(x_{i+1, l^{\prime}}\right)\right\},\left\{x_{i, l}\right\}\right]$ is a basis for $C_{i}(X ; R)$ which conforms to the above recipe. $X_{i}$ is a sublattiice of the reference lattice $\left[\left\{\tilde{h}_{i, k}\right\},\left\{\hat{b}_{i, l^{\prime}}\right\},\left\{x_{i, l}\right\}\right]$ of index $\left|\overline{B_{i}(X ; Z)} / B_{i}(X ; Z)\right|=$ $\theta_{X, i}$. Hence, $D_{i}=\theta_{X, i}$ and the theorem follows.

Returning to the general context of the R-torsion, a chain complex with the above choices $\left\{x_{i, j}\right\},\left\{h_{i, k}\right\}$, there is a real inner product in which these basis elements are orthonormal. Hence, for these inner products the boundary mapping $d_{i+1}$ has an adjoint

$$
d_{i+1}^{\star}: C_{i} \rightarrow C_{i+1}
$$

As before let $\left\{h_{i, k}\right\}$ be a specified basis for the real vector space $C_{i}$. 
As Ray and Singer remark in their foundational paper on analytic torsion [26], there is a formula for the R-torsion $\tau$ in terms of the reduced Laplacian

$$
\tilde{\Delta}_{X, i}:=\left(d_{i+1} \cdot d_{i}^{\star} \mid B_{i}\right): B_{i} \rightarrow B_{i} .
$$

The first part of this relation is a measure, via covolumes of the chosen homology basis: For this purpose, note that one has an orthogonal Hodge decomposition,

$$
C_{i}=\operatorname{Harm}_{i} \oplus\left\{\text { image of } d_{i+1}\right\} \oplus\left\{\text { image of } d_{i-1}^{\star}\right\}
$$

in which $\operatorname{Harm}_{i}=\left\{h \mid d_{i}(h)=0\right.$ and $\left.d_{i-1}^{\star}(h)=0\right\}$ are the i-harmonics. Moreover, $Z_{i}=$ Harm $_{i} \oplus\{$ image of $d\}$ is a orthogonal decomposition. Let $\pi_{i}$ be the orthogonal projection :

$$
\pi_{i}: C_{i} \rightarrow \text { Harm }_{i}
$$

It induces the isomorphism $H_{i}(X ; R)=Z_{i} / B_{i} \cong \operatorname{Harm}_{i}$.

In this manner the specified basis for the integral homology modulo the torsion $\left\{h_{i, k}\right\}$ under the orthogonal projection to $\operatorname{Harm}_{i}$ yields the desired elements $\pi_{i}\left(h_{i, k}\right) \in \operatorname{Harm}_{i}$. They define a $Z$-lattice of full rank which are independent of the lifts chosen for the $\left\{h_{i, k}\right\}$ 's. The subspace $\mathrm{Harm}_{i}$ inherits an inner product from that on $C_{i}$. One defines

$$
\left(\mathcal{H}_{X, i}\right)^{2}=\left[\text { covolume }\left(\text { lattice }\left\{\pi_{i}\left(h_{i, k}\right)\right\} \text { in } C_{i}\right)\right]^{2}=\operatorname{det}\left(\left\{<\pi_{i}\left(h_{i, a}\right), \pi_{i}\left(h_{i, b}\right)>\right\}\right)
$$

as a measure of the size of the combinatorial homology basis.

With these preparations one has:

Theorem 10.2 (Ray and Singer [26]). The R-torsion is given in terms of the covolumes $\mathcal{H}_{i}$ for the i-homologies and the determinant of the reduced Laplacians by

$$
\begin{aligned}
& {\left[\tau\left(C_{\star}(X ; R), \partial_{R},\left\{\text { combinatorial basis for } C_{\star}(X ; R) \text { and } H_{\star}(X ; R)\right)\right]^{2}\right.} \\
& =\prod_{i=0}^{n}\left[\operatorname{det}\left(\tilde{\Delta}_{X, i}\right) \cdot\left(\mathcal{H}_{X, i}\right)^{2}\right]^{(-1)^{i}} .
\end{aligned}
$$

[Note that in Ray and Singer's paper they use the full Laplacian $\Delta=d_{i+1} d_{i}^{\star}+d_{i-1}^{\star} d_{i}$ with corresponding modification of the above formula. They also restrict to the acyclic cases $H_{i}=0$.]

Returning to the cell context and the combinatorial bases for chain and homologies, The formula of Ray and Singer and the above computation yields:

Theorem 10.3. For each finite $C W$ complex, and each choice of skeleton, say $X^{(d)}$,

$$
\begin{aligned}
& {\left[\prod_{k=1}^{d-1} \theta_{X^{(d)}, i}^{(-1)^{i}}\right]^{2}} \\
& =\prod_{i=0}^{d}\left[\operatorname{det}\left(\partial_{i+1} \cdot \partial_{i}^{\star} \mid \text { image of } \partial_{i+1}\right) \cdot\left(\mathcal{H}_{X, i}\right)^{2}\right]^{(-1)^{i}} .
\end{aligned}
$$

This theorem gives a sequence of combinatorial identities since the terms $\operatorname{det}\left(\partial_{i+1} \cdot \partial_{i}^{\star} \mid\right.$ image of $\left.\partial_{i+1}\right)$ are expressed by Lyons's formulae combinatorially.

The papers of M. Catanzaro, V. Chernyak, and J. Klein, 5, 5, 6] also study aspects of the Reidemeister-Fraz torion in the combinatorial context. Their results are complementary to those given here. 


\section{Variant Settings and Examples}

11.1. Weights. One may easily adapt the above discussion to the context of cell complexes with weighted cells. Let $\left\{e_{i, j}\right\}$ be the i-dimensional cells of a cell complex $X$ and $W_{i}$ : $\left\{e_{i, j}\right\} \rightarrow R^{+}$be a positive real valued function. This weighting defines using the cellular basis an isomorphism, $W_{i}: C_{i}(X ; R) \rightarrow C_{i}(X ; R)$ multiplying a cell by its weight. Some familiar examples for graphs are:

- One often introduces multiplicities for the edges.

- For the normalized reduced graph Laplacian a vertex $v$ is weighted by one divided by the degree of the vertex, see F. R. K. Chung [11].

- In the classical Kirchoff circuit setting, the resistances of the wire edges are used as the weights.

The paper of M. Catanzaro, V. Chernyak, and J. Klein [6] generalizes classical results of Kirchhoff on spanning trees, see also 4], to circuits, voltages, resistances, and is written in a weighted higher dimensional setting.

Now all the present results readily extend to the weighted context. There are two formally equivalent methods to carry this out.

In the first method: keeping the the standard cellular inner product, one replaces the mapping $\partial: C_{i+1}(X ; R) \rightarrow C_{i}(X ; R)$ represented by the matrix $A$ and its adjoint $\partial^{\star}: C_{i}(X ; R) \rightarrow$ $C_{i+1}(X ; R)$ represented by the matrix $A$ by the mappings $\mathcal{L}_{W}=\left(W_{i}\right)^{-1 / 2} \partial\left(W_{i+1}\right)^{1 / 2}$ represented by $\left(W_{i}\right)^{-1 / 2} \cdot A \cdot\left(W_{i+1}\right)^{1 / 2}$ and its adjoint $\mathcal{L}^{\star}=\left(W_{i+1}^{1 / 2} \cdot \partial^{\star} \cdot\left(W_{i}\right)^{-1 / 2}\right.$ represented by $\left(W_{i+1}^{1 / 2} \cdot A \cdot\left(W_{i}\right)^{-1 / 2}\right.$. That is, one replaces the reduced Laplacian $\partial \cdot \partial^{\star}$ represented by $A \cdot A^{t}$ by $\mathcal{L} \cdot \mathcal{L}^{\star}$ represented by $\left(\left(W_{i}\right)^{-1 / 2} \cdot A \cdot\left(W_{i+1}\right)^{1 / 2}\right) \cdot\left(\left(W_{i+1}\right)^{1 / 2} \cdot A^{t} \cdot\left(W_{i}\right)^{-1 / 2}\right)=$ $\left(W_{i}\right)^{-1 / 2} \cdot A \cdot W_{i+1} \cdot A^{t} \cdot\left(W_{i}\right)^{-1 / 2}$.

In this approach the operators are changed, but the inner product is left alone. All covolumes are computed for the new operators with the old inner product. With these slight changes, all theorems can be recast in this extended context.

In the second alternative approach: One recognizes that the characteristic polynomial of $\left(W_{i}\right)^{-1 / 2} \cdot A \cdot W_{i+1} \cdot A^{t} \cdot\left(W_{i}\right)^{-1 / 2}$ equals that of $A \cdot W_{i+1} \cdot A^{t} \cdot\left(W_{i}\right)^{-1}$. Now if takes the adjoint of $\partial$ represented by $A$ with respect to the modified inner product $<..\rangle_{W}$ given by $<e_{i, j}, e_{i, k}>_{W}=\delta_{j, k} W\left(e_{i, j}\right)$ then the adjoint, called here $\partial^{\star, W}$ is represented by $W_{i+1} \cdot A^{t} \cdot\left(W_{i}\right)^{-1}$. That is, one may rephrase the above theorem in terms of $\partial \cdot \partial^{\star, W}$ by computing the covolumes utilizing the new inner product $<.,>_{W}$ but with $\partial$ unchanged.

All the results of the present paper can be readily extended to these weighted settings. For example, the analogue of Theorem 2.1 is

Theorem 11.1. Let $X$ be a d-dimensional cell complex.

The $k^{\text {th }}$ elementary symmetric polynomial in the eigenvalues of the reduced weighted Laplacian $\mathcal{L} \cdot \mathcal{L}^{\star}$ are combinatorially expressed in term of covolumes of lattices with respect to the weighted metric $<.,>_{W}$ as follows:

$$
\begin{aligned}
& \sigma_{k}(\tilde{\Delta})=\left[\text { covolume }\left(\text { lattice image } \partial_{R}^{\star}\left(C_{d}(X ; Z)\right) \subset C_{d+1}(X ; Z) \text { in } C_{d+1}(X ; R)\right)\right]^{2}
\end{aligned}
$$

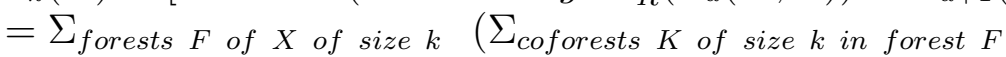

$$
\begin{aligned}
& \text { [covolume(lattice image } \left.\left.\left.B_{d-1}(F ; Z) \rightarrow C_{d-1}(K ; R)\right)\right]^{2}\right)
\end{aligned}
$$


where the mapping $B_{d-1}(F ; Z) \rightarrow C_{d-1}(K ; R)$ proceeds by inclusion into $C_{d-1}(F ; R)$ and then restriction to $C_{d-1}(K ; R)$

Here if there are no forests, or coforests of the required type, then that term is to be interpreted as zero.

And the analogue of the corollary of the Trent type theorem in the weighted setting is: Let all covolumes in the next statement be computed with respect to the weighted inner product $<., .>_{W}$. Then an inductive calculation of the covolumes of $Z_{i}(X ; Z) \subset C_{i}(X ; R)$ computed with respect to the weighted inner product is:

\section{Corollary 11.2.}

[covolume of lattice $\left.\left.Z_{d}(X ; Z) \subset C_{d}(X ; R)\right)\right]^{2}$ $=\left[\right.$ covolume of lattice $\left.\left.Z_{d-1}(X ; Z) \subset C_{d-1}(X ; R)\right)\right]^{2}$. $\left(\left(\mathcal{H}_{k, X}\right)^{2} \cdot\left[\text { covolume }\left(\overline{B_{k}(X ; Z)} \subset C_{k}(X ; R)\right)\right]^{2} \cdot\left(\Sigma_{T \in \mathcal{T}_{k+1}}\left(\frac{\theta_{T, k}}{\theta_{X, k}}\right)^{2}\right)\right.$.

We leave as an exercise to the reader the rewriting of the other theorems above into this enlarged setting.

Remark: Likewise, all the results of this paper can also be rewritten to encompass weights which are allowed to be non-zero real numbers (or even non-zero complex numbers), e.g., using the normal principal values for square roots. An interesting example of positive and negative edge weights \pm 1 occurs in the work of K. Marasugi [23] on polynomial invariants of knots.

11.2. Twisted Coefficients. Historically, Reidemeiser-Franz torsion was introduced as an invariant of finite cell complexes with $U(1)$ representations of the fundamental group, equivalently flat $U(1)$ bundes. The definitions were later extended to arbitrary unitary representations.

From this point of view the above combinatorial results are in the setting of the trivial $U(1)$ representation. As also done for some results on spanning trees by M. Catanzaro, V. Chernyak, and J. Klein [5], in this section the above combinatorial results are extended to $U(1)$ representations.

Given a representation $\rho: \pi_{1}(X) \rightarrow U(1)$ of the fundamental group of a connected cell complex $X$ into $U(1)$, one may form the chains with values in $C$ twisted by the flat representation $\rho, C_{\star}\left(X ; E_{\rho}\right)$ together with it boundary mapping

$$
\partial_{\rho}: C_{i+1}\left(X ; E_{\rho}\right) \rightarrow C_{i}\left(X, E_{\rho}\right) .
$$

Since $\rho$ is unitary, the standard Hermitian pairing $\langle z, w>\mapsto z \bar{w}$ on $C$ extends to a Hermitian inner product on $\mathrm{i}$-chain with values in the local system $E_{\rho}$ :

$$
<.,>: C_{i}\left(x ; E_{\rho}\right) \times C_{i}\left(X ; E_{\rho}\right) \rightarrow C
$$

Hence, the adjoint $\partial_{\rho}^{\star} ; C_{i}\left(X ; E_{\rho}\right) \rightarrow C_{i+1}\left(X ; E_{\rho}\right)$ is well defined.

Now suppose that $X$ is a d-dimensional cell complex with i-dimensional cells $\left\{e_{i, j}\right\}$. One may consider the one dimensional vector space $C_{i}\left(X ; E_{\rho}\right)$ of chain with support in that cell, say

$$
V\left[e_{i, j}\right]=\left\{c \in C_{i}\left(X ; E_{\rho}\right) \mid c \text { vanishes on cells not equal to } e_{i, j}\right\}
$$


ENUMERATIVE COMBINATORICS OF SIMPLICIAL AND CELL COMPLEXES: KIRCHHOFF AND TRENT TYPE THEOREN\&g

This provides a canonical orthogonal direct sum decomposition of the $i-$ chains :

$$
C_{i}\left(X ; E_{\rho}\right)=\bigoplus_{j} V\left[e_{i, j}\right]
$$

For example, for any subcomplex $S \subset X$ containing say $k$ cells of dimension $d$ and all of the $d-1$ skeleton, one may consider the vector space of dimension $k$ of $C_{d}\left(X ; E_{\rho}\right)$ define by

$$
V_{i}[S]=\left\{c \in C_{i}\left(X ; E_{\rho}\right) \mid \text { c vanishes on cells not equal to } e_{i, j}\right\}=\bigoplus_{e_{d, j}}^{\bigoplus \text { a d-cell of } S} V\left[e_{d, j}\right] .
$$

Naturally there are boundary mapping $(\partial \mid S): V_{d}[S] \rightarrow V_{d-1}[S]$.

Now picking bases elements of length one for the one dimensional vector spaces $V\left[e_{d, j}\right]$ and $V\left[e_{d-1, k}\right]$ the boundary mapping

$$
\partial: C_{d}\left(X ; E_{\rho}\right) \rightarrow C_{d-1}\left(X ; E_{\rho}\right)
$$

is represented by a matrix, say $A$, with rows and columns indexed by the $d$-1-cells of $X$ and the $d-1$ cells of $X$ respectively, or alternatively the one dimensional blocks, $\left.V\left[e_{d-1, k}\right], V\left[e_{d, j}\right]\right]$, respectively.

Consequently, the algebraic lemmas 3.1,3.2 apply, giving by the usual method

$$
\begin{aligned}
& \sigma_{k}\left(\partial_{\rho} \cdot \partial_{\rho}^{\star}: C_{d-1}\left(X ; E_{\rho}\right) \rightarrow C_{d-1}\left(X ; E_{\rho}\right)\right) \\
& =\sigma_{k}\left(\partial_{\rho}^{\star} \cdot \partial_{\rho}: C_{d}\left(X ; E_{\rho}\right) \rightarrow C_{d}\left(X ; E_{\rho}\right)\right)
\end{aligned}
$$

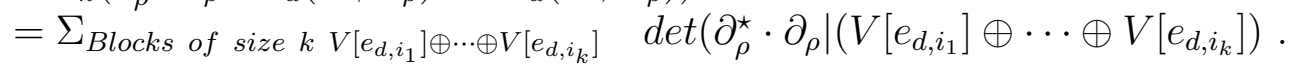

Analogous to the above, define a subcomplex $F \subset X$ to be a d-dimensional forest of size $k$ for $\rho$ if $F$ includes the $d-1$ skeleton of $X, F$ contains exactly $k$ cells of dimension $d$ and the boundary mapping

$$
(\partial \mid S): C_{d}\left(S ; E_{\rho}\right) \rightarrow C_{d-1}\left(S ; E_{\rho}\right)
$$

is one to one.

By application of the algebraic identity above, one gets the analogue of theorem 2.1 in this twisted context:

$$
\begin{aligned}
& \sigma_{k}\left(\partial_{\rho} \cdot \partial_{\rho}^{\star}: C_{d-1}\left(X ; E_{\rho}\right) \rightarrow C_{d-1}\left(X ; E_{\rho}\right)\right) \\
& =\Sigma_{d-\text { dimensional forests } F \text { of size } k} \operatorname{det}\left(A_{F}^{\star} \cdot A_{F}\right)
\end{aligned}
$$

where $A_{F}$ is the composite $C_{d}\left(F ; E_{\rho}\right) \stackrel{\partial \mid S}{\rightarrow} C_{d-1}\left(S ; E_{\rho}\right) \subset C_{d-1}\left(X ; E_{\rho}\right)$.

Of course, there are analogous theorems about forests and coforests and their dual notions.

As mentioned above, the spanning tree case of coefficients in a flat complex line bundle where $k=\operatorname{dim} B_{d-1}\left(X ; E_{\rho}\right)$, is discussed in M. Catanzaro, V. Chernyak, and J. Klein [5] and there related directly to circuit theory.

11.3. Manifolds. When $K$ is a triangulation of a closed n-manifold, one may introduce the dual cell subdivision, $K^{\star}$ whose $(n-i)$-cells, $D\left(e_{i, j}\right)$, are indexed by the baricenters, say $\hat{\sigma}_{i, j}$ of the i-dimensional simplices $\sigma_{i, j}$. The dual cell $D\left(\sigma_{i, j}\right)$ consists of the sets of simplices $<\hat{\sigma}_{i, j_{1}}, \hat{\sigma}_{i+1, j_{2}}, \cdots, \hat{\sigma}_{n, j_{n-i+1}}>$ of the baricentric subdivision of $K$ where the baricenters correspond to an increasing flag of simplicies of $K$ starting with $\sigma_{i, j}=\sigma_{i, j_{1}}$. That is, the simplicies $\sigma_{i+k-1, j_{k}}$ of $K$ are of dimension $i-1+k$, with $k=1, \cdots, n-i+1$ and $\sigma_{i, j}=$ 
$\sigma_{i, j_{1}} \subset \sigma_{i+1, j_{2}} \subset \cdots \subset \sigma_{n, j_{n-i+1}}$. The bijection $\sigma_{i, j} \mapsto D\left(\sigma_{i, j}\right)$ establishes an isomorphism of chains

$$
C_{i}(K ; Z) \cong C_{n-i}(K ; Z) \text { and } C_{i}(K ; R) \cong C_{n-i}(K ; R)
$$

under which the boundary $\partial$ of $K$ and its dual $\partial^{\star}$, the "coboundary" for the cellular inner product on $K$, are identified with the coboundary for $K^{\star}$ and the boundary for $K^{\star}$, respectively.

Hence, in this special context, forests of $K$ correspond to dual forests of $K^{\star}$, coforests of $K$ correspond to dual coforests of $K^{\star}$, etc.

\section{Enumerative combinatorics of the simplex following Kalai [14]}

Let $\Delta_{n-1}$ denote the standard $n-1$ simplex with $n$ vertices, say $x_{1}, \cdots, x_{n}$ in this order. Regard $\Delta_{n-1}$ as the join

$$
\Delta_{n-1}=x_{1} \star \Delta_{n-2}
$$

where $\Delta_{n-2}$ is the standard $n-2$ simplex with vertices $x_{2}, \cdots, x_{n}$. For a simplex, say $s$ in $\Delta_{n-1}$, let $s$ have its inherited orientation from this choice of ordering; for emphasis, denote the oriented simplex $\vec{s}$. As $s$ ranges over the $j$-simplices of $\Delta_{n-1}$, respectively $\Delta_{n-2}$, the elements $1 \cdot \vec{s}$ provide an integral basis for the $j$-chains, $C_{j}\left(\Delta_{n-1} ; Z\right), C_{j}\left(\Delta_{n-2} ; Z\right)$. With respect to these cellular choices of basis, the boundary maps

$$
\partial_{\Delta_{n-1}}: C_{k}\left(\Delta_{n-1} ; Z\right) \rightarrow C_{k-1}\left(\Delta_{n-1} ; Z\right) \text { and } \partial_{\Delta_{n-2}}: C_{k}\left(\Delta_{n-2} ; Z\right) \rightarrow C_{k-1}\left(\Delta_{n-2} ; Z\right)
$$

are represented by the respective incidence matrices, and have adjoints, with respect to the cellular bases, $\partial_{\Delta_{n-1}}^{\star}$ and $\partial_{\Delta_{n-2}}^{\star}$ represented by the transposes of these incidence matrices respectively.

Note that the integral d-chains, $C_{d}\left(\Delta_{n-1} ; Z\right)$, for $d \leq n-1$ have a spanning tree consisting of all $d$-cells, say $s$, containing the initial vertex $x_{1}$. Moreover, associated to this spanning tree the integral d-cycles, $Z_{d}\left(\Delta_{n-1} ; Z\right)$ for $d<n-1$ have a basis consisting of the boundaries $\partial\left(x_{1} \star t\right)$ where $t$ ranges over the d-cells in $\Delta_{n-2}$, that is the d-cells not containing $x_{1}$. Consequently, the above theorems apply in this context.

Now consider the standard cellular inclusion :

$$
i: C_{k-1}\left(\Delta_{n-2} ; Z\right) \rightarrow C_{k-1}\left(\Delta_{n-1} ; Z\right)
$$

In the cellular basis, the adjoint is just the cellular restriction mapping

$$
C_{k-1}\left(\Delta_{n-1} ; Z\right) \stackrel{\text { restriction }}{\rightarrow}=i^{\star} C_{k-1}\left(\Delta_{n-2} ; Z\right) .
$$

Following, Kalai, consider the composite :

$$
i^{\star} \cdot \partial_{\Delta_{n-1}}: C_{k}\left(\Delta_{n-1} ; Z\right) \rightarrow C_{k-1}\left(\Delta_{n-2} ; Z\right)
$$

It is represented by the restricted incidence matrix, say $I_{r}^{k}$, obtained by deleting the rows corresponding to $k-1$ simplices not in $\Delta_{n-2}$. In these terms, one of Kalai's results takes the following form: 
ENUMERATIVE COMBINATORICS OF SIMPLICIAL AND CELL COMPLEXES: KIRCHHOFF AND TRENT TYPE THEOREN\$\$

Theorem 12.1 (Kalai ). The composite

$$
\left(i^{\star} \cdot \partial_{\Delta_{n-1}}\right) \cdot\left(i^{\star} \cdot \partial_{\Delta_{n-1}}\right)^{\star}: C_{k-1}\left(\Delta_{n-2} ; Z\right) \rightarrow C_{k-1}\left(\Delta_{n-2} ; Z\right)
$$

which is represented by $I_{r}^{k} \cdot\left(I_{r}^{k}\right)^{t}$, has eigenvalues 1 and $n$ with respective multiplicities $\left(\begin{array}{l}n-2 \\ k-1\end{array}\right)$ and $\left(\begin{array}{c}n-2 \\ k\end{array}\right)$.

Note that, as expected, the sum of these multiplicities is $\left(\begin{array}{c}n-1 \\ k\end{array}\right)=\operatorname{rank} C_{k-1}\left(\Delta_{n-2} ; Z\right)$.

The result easily gives the eigenvalues and their multiplicities of the Kirchhoff matrix representing the combinatorial Laplacian

$$
\partial_{\Delta_{n-2}} \cdot \partial_{\Delta_{n-2}}^{\star}: C_{k-1}\left(\Delta_{n-2}: Z\right) \rightarrow C_{k-1}\left(\Delta_{n-2}: Z\right)
$$

as follows:

For $s$ a $k-1$ simplex in $\Delta_{n-2}$, one computes :

$$
\begin{aligned}
& \left(i^{\star} \cdot \partial_{\Delta_{n-1}}\right) \cdot\left(i^{\star} \cdot \partial_{\Delta_{n-1}}\right)^{\star}(1 \cdot \vec{s})=\left(i^{\star} \cdot \partial_{\Delta_{n-1}}\right) \cdot \partial_{\Delta_{n-1}}^{\star} \cdot i(1 \cdot \vec{s})=\left(i^{\star} \cdot \partial_{\Delta_{n-1}}\right) \cdot\left(\partial_{\Delta_{n-1}}^{\star}(1 \cdot \vec{s})\right) \\
& =\left(i^{\star} \cdot \partial_{\Delta_{n-1}}\right) \cdot\left(1 \cdot x_{1} \star \vec{s}+i \cdot\left(\partial_{\Delta_{n-2}}^{\star}(1 \cdot \vec{s})\right)\right)=i^{\star}\left(1 \cdot \vec{s}-x_{1} \star \partial_{\Delta_{n-2}}^{\star}(1 \cdot \vec{s})+\partial_{\Delta_{n-2}}\left(\partial_{\Delta_{n-2}}(1 \cdot \vec{s})\right)\right) \\
& =1 \cdot \vec{s}+\partial_{\Delta_{n-2}}\left(\partial_{\Delta_{n-2}}^{\star}(1 \cdot \vec{s})\right.
\end{aligned}
$$

That is, one sees that

$$
\left(i^{\star} \cdot \partial_{\Delta_{n-1}}\right) \cdot\left(i^{\star} \cdot \partial_{\Delta_{n-1}}\right)^{\star}=I d+\partial_{\Delta_{n-2}}\left(\partial_{\Delta_{n-2}}^{\star}\right)
$$

yielding the following corollary to Kalai's result. Let $B d^{k}$ be the matrix representing the boundary mapping $\partial_{\Delta_{n-2}}: C_{k}\left(\Delta_{n-2}: Z\right) \rightarrow C_{k-1}\left(\Delta_{n-2}: Z\right)$.

Corollary 12.2. The combinatorial Laplacian

$$
\partial_{\Delta_{n-2}} \cdot \partial_{\Delta_{n-2}}^{\star}: C_{k-1}\left(\Delta_{n-2}: Z\right) \rightarrow C_{k-1}\left(\Delta_{n-2}: Z\right)
$$

is represented by the matrix $B d^{k} \cdot\left(B d^{k}\right)^{t}$ and has eigenvalues 0 and $n-1$ with respective multiplicities $\left(\begin{array}{l}n-2 \\ k-1\end{array}\right)$ and $\left(\begin{array}{c}n-2 \\ k\end{array}\right)$.

Now there is a natural integral and geometric basis for the integral $k-1$ cycles of $\Delta_{n-1}$, $Z_{k-1}\left(\Delta_{n-1} ; Z\right)$, provided by the following map from $k-1$ chains on $\Delta_{n-2}$ to $k-1$ cycles which is an isomorphism:

$$
\begin{aligned}
& \Phi: C_{k-1}\left(\Delta_{n-2} ; Z\right) \rightarrow Z_{k-1}\left(\Delta_{n-1} ; Z\right) \\
& 1 \cdot \vec{s} \mapsto \partial_{\Delta_{n-1}}\left(x_{1} \star \vec{s}\right) \text { for } s \text { a } k \text { simplex in } \Delta_{n-2}
\end{aligned}
$$

Since $\partial_{\Delta_{n-1}}\left(x_{1} \star \vec{s}\right)=1 \cdot \vec{s}-x_{1} \star \partial_{\Delta_{n-2}}(1 \cdot \vec{s})$, the composite $i^{\star} \Phi(1 \cdot \vec{s})$ equal just $1 \cdot \vec{s}$. That is, $i^{\star} \cdot \Phi=$ Identity. Hence, $\Phi$ is one to one.

Next given a $k-1$ cycle, $z \in Z_{k-1}\left(\Delta_{n-1} ; Z\right)$, then $z=\Phi\left(i^{\star}(z)\right)+\left(z-\Phi\left(i^{\star}(z)\right)\right.$ where $i^{\star}\left(z-\Phi\left(i^{\star}(z)\right)=0\right.$. Hence, $\left(z-\Phi\left(i^{\star}(z)\right)=x_{1} \star c\right.$ for some $k-2$ chain $c$ supported in $\Delta_{n-2}$. But then $0=i^{\star}\left(\partial_{\Delta_{n-1}}\left(z-\Phi\left(i^{\star}(z)\right)\right)=i^{\star}\left(\partial_{\Delta_{n-1}}\left(x_{1} \star c\right)\right)=i^{\star}\left(c-x_{1} \star \partial_{\Delta_{n-2}}(c)\right)=c\right.$. This proves that $c=0$ and so that $z=\Phi\left(i^{\star}(z)\right)$ when $z$ is an integral $k-1$ cycle.

In toto, this shows that $\Phi$ is an isomorphism.

In particular, the cellular basis for $C_{k-1}\left(\Delta_{n-2}, ; Z\right)$ gives the geometric basis $\{\Phi(1 \cdot \vec{s})\}$ for the $k-1$ cycles. As expected, using the Kalai result again, one may use this method to find the eigenvalues and multiplicities of the mesh matrix for $k-1$ cycles. 
Let $J^{k-1}$ represent the inclusion $Z_{k-1}\left(\Delta_{n-1} ; Z\right) \subset C_{k-1}\left(\Delta_{n-1} ; Z\right)$ with respect to the geometrical basis $\{\Phi(s)\}$ of $k-1$ cycles and the cellular basis of $k-1$ chains. That is, $J^{k-1}$ represents the composite $C_{k-1}\left(\Delta_{n-2} ; Z\right) \stackrel{\Phi}{\rightarrow} Z_{k-1}\left(\Delta_{n-1} ; Z\right) \stackrel{i}{\rightarrow} C_{k-1}\left(\Delta_{n-1} ; Z\right)$ with respect to the cellular bases.

Corollary 12.3. For the integral basis of $Z_{k-1}\left(\Delta_{n-1}, Z\right)$ provided by the geometric integral basis $\Phi(1 \cdot \vec{s})$ where $s$ ranges over the $k-1$ simplices of $\Delta_{n-2}$, the mesh matrix for cycles representing the pairing

$$
Z_{k-1}\left(\Delta_{n-1} ; Z\right) \times Z_{k-1}\left(\Delta_{n-1} ; Z\right) \rightarrow Z
$$

with respect to this basis, or equivalently of the matrix $J^{k-1}\left(J^{k-1}\right)^{t}$, has eigenvalues only equal to $n$ and 1 with respective multiplicities $\left(\begin{array}{c}n-2 \\ k-1\end{array}\right)$ and $\left(\begin{array}{c}n-2 \\ k\end{array}\right)$.

Since $Z_{d}\left(\Delta_{n}: Z\right)=B_{d}\left(\Delta_{n} ; Z\right)$, the cycle and boundary mesh matrices are identical in this case.

To prove this it suffices to compute and simplify the entries with $s, s^{\#}$ two $k-1$ simplicies in $\Delta_{n-2}$ :

$$
\begin{aligned}
& <\Phi(1 \cdot \vec{s}), \Phi\left(1 \cdot \vec{s}^{\#}>=<\partial_{\Delta}\left(x_{1} \star \vec{s}\right), \partial_{\Delta}\left(x_{1} \star \vec{s}^{\#}\right)>\right. \\
& =<1 \cdot \vec{s}-x_{1} \star \partial_{\Delta_{n-2}}(\vec{s}), 1 \cdot \vec{s}^{\#}-x_{1} \star \partial_{\Delta_{n-2}}\left(\vec{s}^{\#}\right)>=\delta_{s, s}^{\#}+<\partial_{\Delta_{n-2}}(\vec{s}), \partial_{\Delta_{n-2}}\left(\vec{s}^{\#}\right)> \\
& =\delta_{s, s}+<\vec{s}, \partial_{\Delta_{n-2}}^{\star} \partial_{\Delta_{n-2}}\left(\vec{s}^{\#}\right)>
\end{aligned}
$$

Now by Lemma 3.2 the non-zero eigenvalues of $\partial_{\Delta_{n-2}}^{\star} \partial_{\Delta_{n-2}}$ acting on $C_{k-1}\left(\Delta_{n-2} ; Z\right)$ are the same as that of the combinatorial Laplacian, or Kirchhoff map, $\partial_{\Delta_{n-2}} \partial_{\Delta_{n-2}}^{\star}$ acting on $C_{k-2}\left(\Delta_{n-2} ; Z\right)$. By corollary 12.2, this last has a single non vanishing eigenvalue, nameby $n-1$, of multiplicity $\left(\begin{array}{l}n-2 \\ k-1\end{array}\right)$. Hence, the mesh matrix above has an eigenvalue $n$ of multiplicity $\left(\begin{array}{l}n-2 \\ k-1\end{array}\right)$ and eigenvalue 1 of multiplicity $\left(\begin{array}{c}n-1 \\ k\end{array}\right)-\left(\begin{array}{c}n-2 \\ k-1\end{array}\right)=\left(\begin{array}{c}n-2 \\ k\end{array}\right)$ as claimed.

Kalai [14] has given an additional refinement of his result recorded here in theorem 12.1. For each vertex $x_{j}$ introduce a real positive indeterminant, say $a_{j}>0$. Introduce a weight for each face $f=<x_{i_{1}}, x_{i_{2}}, \cdots, x_{i_{l}}>$ with $w(f)$ equal to the product $a_{i_{1}} \cdot a_{i_{2}} \cdots a_{i_{l}}$ of the corresponding indeterminants of the vertices of the face $f$. This defines an inner product on each of the $j$ chains for which the elements $\{1 \cdot f / \sqrt{w(f)}\}$ are an orthonormal basis. These are called the normalized cellular basis, Let $D(k, m)$ be the diagonal self mapping of the $k$ chains on $\Delta_{m}$ which multiplies a $k$ face $f$ by $w(f)$. For convenience, also let $D(k, m)$ also denote the diagonal matrix which represents $D(k, m)$ in the cellular basis.

Now the Kalai mapping $i^{\star} \cdot \partial_{\Delta_{n-1}}: C_{k}\left(\Delta_{n-1} ; Z\right) \rightarrow C_{k-1}\left(\Delta_{n-2} ; Z\right)$ with respect to this new normalized cellular basis gives a reduced incidence matrix, say $\left(I_{r}^{k}\right)^{\#}$ which is related to the original reduced incidence matrix $I_{r}^{k}$ by

$$
\left(I_{r}^{k}\right)^{\#}=D(k-1, n-2)^{-1 / 2} I_{r}^{k} \cdot D(k, n-1)^{1 / 2} .
$$

Kalai's extension states that the matrix

$$
\left(I_{r}^{k}\right)^{\#} \cdot\left(\left(I_{r}^{k}\right)^{\#}\right)^{t}=D(k-1, n-2)^{-1 / 2} I_{r}^{k} \cdot D(k, n-1)\left(I_{r}^{k}\right)^{t} \cdot D(k, n-2)^{-1 / 2}
$$

has eigenvalues $a_{1}$ and $\sum_{j=1}^{n} a_{j}$ with multiplicities $\left(\begin{array}{c}n-2 \\ k-1\end{array}\right)$ and $\left(\begin{array}{c}n-2 \\ k\end{array}\right)$ [14]. 
Correspondingly, the boundary mapping $\partial_{\Delta_{n-2}}: C_{k-1}\left(\Delta_{n-2}: Z\right) \rightarrow C_{k-2}\left(\Delta_{n-2}: Z\right)$ with respect to the new normalized cellular basis is a matrix, say $\left(B d^{k-1}\right)^{\#}$. It is related to the standard boundary mapping $B d^{k-1}$ by

$$
\left(B d^{k-1}\right)^{\#}=D(k-1, n-2)^{-1 / 2}\left(B d^{k-1}\right) D(k, n-1)^{1 / 2} .
$$

The normalized Kirchhoff mapping is then represented by

$$
\left(B d^{k-1}\right)^{\#}\left(\left(B d^{k-1}\right)^{\#}\right)^{t}=D(k-1, n-2)^{-1 / 2}\left(B d^{k-1}\right) D(k, n-1)\left(B d^{k-1}\right)^{t} D(k-1, n-2)^{-1 / 2} .
$$

A slight extension of the above argument shows that this matrix has eigenvalues 0 and $\begin{array}{lll}\sum_{j=1}^{n-1} & a_{j}\end{array}$ with multiplicities with respective multiplicities $\left(\begin{array}{c}n-2 \\ k-1\end{array}\right)$ and $\left(\begin{array}{c}n-2 \\ k\end{array}\right)$. This follows from Kalai's results by the method used in the unnormalized case above.

Correspondingly, under the identification $\Phi: C_{k-1}\left(\Delta_{n-2} ; Z\right) \rightarrow Z_{k-1}\left(\Delta_{n-1} ; Z\right)$, one has a bilinear "mesh" pairing

$$
C_{k-1}\left(\Delta_{n-2} ; Z\right) \times C_{k-1}\left(\Delta_{n-2} ; Z\right) \rightarrow Z_{k-1}\left(\Delta_{n-1} ; Z\right) \times Z_{k-1}\left(\Delta_{n-1} ; Z \rightarrow R\right.
$$

where the last bilinear pairing used the chosen weights $w(f)$.

For the choice of normalized cellular basis for $C_{k-1}\left(\Delta_{n-2} ; Z\right)$, it is easily seen, by the method above, that this matrix has eigenvalues $a_{1}$ and $\sum_{j=1}^{n-1} a_{j}$ of respective multiplicities $\left(\begin{array}{l}n-2 \\ k-1\end{array}\right)$ and $\left(\begin{array}{c}n-2 \\ k\end{array}\right)$. This follows from Kalai's results by the method used in the unnormalized case above.

Extending in several directions some of the formulae of Kalai, the complete calculations in this section of the eigenvalues of the Kirchhoff matrix and of the two mesh matrices in each dimension of simplices, yield, by results developed in the preceding sections, many combinatorial identities involving appropriate subobjects of simplices for each of the coefficients of the corresponding characteristic polynomials.

\section{REFERENCES}

[1] R. M. Adin, Counting Colorfull Multi-dimensional Trees, Combinatorica, vol. 12 (1992), pp. 247-260

[2] N. L. Biggs, Algebraic Graph Theory, Cambridge University Press, 1960.

[3] B. Bollobás, Modern Graph Theory, Graduate Texts in Mathematics, vol 184, Springer-Verlag, New York, 1998.

[4] P. R. Bryant, Graph Theory applied to electrical networks in F. Harary (ed.), Graph Theory and theoretical physics (1967), Academic Press, N.Y., pp 111-137.

[5] M. Catanzaro, V. Chernyak, and J. Klein, On Kirchhoffs theorems with coefficients in a line bundle, Homology, Homotopy and Applications, vol. 15 no. 2 (2013), 267280.

[6] M. Catanzaro, V. Chernyak, and J. Klein, Kirchhoff's Theorems in Higher Dimensions and Reidemeister Torsion, arXiv:1206.6783 [math.AT].

[7] A. Cayley, A theorem on trees, Quart. J. Math. 23 (1889), pp. 376-378.

[8] S. Chaiken and D. Kleitman, Matrix Tree Theorems, J. of Combinatorial Theory, Series A, 24 (1978), pp. $377-381$.

[9] S. Chaiken, A Combinatorial Proof of the All-Minors Tree Theorem, SIAM J. Alge. Disc. Methods, 3 (1982), pp. 319-329.

[10] W. K. Chen, On vector spaces associated with a graph, SIAM Journal on Applied Mathematics , 20 (1971), pp. 526-529.

[11] F. R. K. Chung, Spectral Graph Theory, American Mathematical Society, 1997.

[12] A. Duval, C. Klivans, and J. Martin, Simplicial Matrix Tree Theorems, Trans. Amer. Math. Soc., vol 361 (2009), pp. 6073-6114.

[13] W. Franz, Ueber die Torsion einer Ueberdeckung, J. Reine Angew. Math., 173 (1935), pp. 245254.

[14] G. Kalai, Enumeration of Q-acyclic Simplicial Complexes, Israel J. Math., vol 45 (1983), pp. 337-351.

[15] R. Kenyon, The Asymptotic Determinant of the Discrete Laplacian, Acta Math., vol 185 (2000), pp. 239-286.

[16] R. Kenyon, Spanning forests and the vector bundle laplacian, The Annals of Probability, 39 no. 5 (2011), pp. 1983-2017.

[17] G. Kirchhoff, Uber die Auflüung der Gleichungen, auf welche man bei der Untersuchung der linearen Verteilung galvanischer Strome gerfuhrt wird, Poggendorfs Ann. Phys. Chem., vol. 72 (1847), pp. 497-508; English translation, IRE Trans. Circuit Theory, vol. 5 (1958), pp. 4-8.

[18] R. Lyons, Random Complexes and $l^{2}$-Betti numbers,, J. Topol. Anal. vol. 1 (2009), pp. 153-175. 
[19] S. B. Maurer, Matrix Generalizations of Some Theorems on Trees, Cycles and Cocycles in Graphs, SIAM Journal on Applied Mathematics, Vol. 30 (1976), pp. 143-148.

[20] J. W. Milnor, A duality theorem for Reidemeister torsion, Ann. of Math., 76 (1962), pp. 137-147.

[21] J. W. Milnor, Whitehead torsion, Bull. Amer. Math. Soc., 72 (1966), pp. 358-426.

[22] J.W. Moon, Counting Labeled Trees, Canadian Mathematical Congress, 1970.

[23] K. Murasugi, On Invariants of Graphs wiht Applications to Knot Theory, Trans. of Amer. Math. Soc., vol 314, \# 1 (1989), pp. $1-49$.

[24] A. Nerode and H. Shank, An algebraic proof of Kirchhoffs network theorem, Amer. Math. Monthly, 68 (1961),pp. $244-247$.

[25] A. Peterson, Enumerating Spanning Trees in Simplicial Complexes, Upsala University preprint, May 18,2009.

[26] Ray, D. B.; Singer, I. M. (1971), R-torsion and the Laplacian on Riemannian manifolds, Advances in Math. 7 (2): 145-210,

[27] K. Reidemeister (1935) Homotopieringe und Linsenrume, Abh. Math. Sem. Univ. Hamburg 11: (1935) pp. 102109.

[28] H. Shank, Graph property recognition machines, Math. Systems Theory, (1971) 5 , pp. 45-49.

[29] H. Trent, A note on the enumeration and listing of all maximal trees of a connected linear graph, Proc. Nat. Acad. Sci. U.S.A., 40 (1954), pp. 1004-1007.

[30] H. M. Trent, Isomorphisms between Oriented Graphs and Lumped Physical Systesm, J. Acoustical Society of America, 27, \#3, (1955), pp. 500-527.

[31] W. Tutte, On the spanning trees of self-dual maps, in Second International Conference on Combinatorial Mathematics (A. Gewirtz andL/V. Quintas, eds.), The New York Academy of Science, 1979, pp.540-548.

[32] , H. Weyl, Classical Groups, Their Invariants and Representations, Princeton University Press (1939).

[33] H. Whitney, The abstract properties of linear independence, Amer. J. Math., 57 (1935), pp. 509-533.

Sylvain E. Cappell, Courant Institute of Mathematical Sciences, New York University. cappell@cims.nyu.edu

Edward Y. Miller, Mathematics Department, Tandon School of Engineering of New York University. em1613@nyu.edu 